\title{
Sharp Inequalities for the Haar System and Fourier Multipliers
}

\author{
Adam Osȩkowski \\ Department of Mathematics, Informatics and Mechanics, University of Warsaw, Banacha 2, 02-097 Warsaw, Poland
}

Correspondence should be addressed to Adam Osękowski; ados@mimuw.edu.pl

Received 27 May 2013; Accepted 30 September 2013

Academic Editor: Kehe Zhu

Copyright (c) 2013 Adam Osękowski. This is an open access article distributed under the Creative Commons Attribution License, which permits unrestricted use, distribution, and reproduction in any medium, provided the original work is properly cited.

A classical result of Paley and Marcinkiewicz asserts that the Haar system $h=\left(h_{k}\right)_{k \geq 0}$ on $[0,1]$ forms an unconditional basis of $L^{p}(0,1)$ provided $1<p<\infty$. That is, if $\mathscr{P}_{J}$ denotes the projection onto the subspace generated by $\left(h_{j}\right)_{j \in J}(J$ is an arbitrary subset of $\mathbb{N})$, then $\left\|\mathscr{P}_{J}\right\|_{L^{p}(0,1) \rightarrow L^{p}(0,1)} \leq \beta_{p}$ for some universal constant $\beta_{p}$ depending only on $p$. The purpose of this paper is to study related restricted weak-type bounds for the projections $\mathscr{P}_{J}$. Specifically, for any $1 \leq p<\infty$ we identify the best constant $C_{p}$ such that $\left\|\mathscr{P}_{J} \chi_{A}\right\|_{L^{p, \infty}(0,1)} \leq C_{p}\left\|\chi_{A}\right\|_{L^{p}(0,1)}$ for every $J \subseteq \mathbb{N}$ and any Borel subset $A$ of $[0,1]$. In fact, we prove this result in the more general setting of continuous-time martingales. As an application, a related estimate for a large class of Fourier multipliers is established.

\section{Introduction}

Our motivation comes from a very natural question about $h=$ $\left(h_{n}\right)_{n \geq 0}$, the Haar system on $[0,1]$. Recall that this collection of functions is given by

$$
\begin{gathered}
h_{0}=[0,1), \\
h_{1}=\left[0, \frac{1}{2}\right)-\left[\frac{1}{2}, 1\right), \\
h_{2}=\left[0, \frac{1}{4}\right)-\left[\frac{1}{4}, \frac{1}{2}\right), \\
h_{3}=\left[\frac{1}{2}, \frac{3}{4}\right)-\left[\frac{3}{4}, 1\right), \\
h_{4}=\left[0, \frac{1}{8}\right)-\left[\frac{1}{8}, \frac{1}{4}\right), \\
h_{5}=\left[\frac{1}{4}, \frac{3}{8}\right)-\left[\frac{3}{8}, \frac{1}{2}\right), \\
h_{6}=\left[\frac{1}{2}, \frac{5}{8}\right)-\left[\frac{5}{8}, \frac{3}{4}\right), \\
h_{7}=\left[\frac{3}{4}, \frac{7}{8}\right)-\left[\frac{7}{8}, 1\right),
\end{gathered}
$$

and so on. Here we have identified a set with its indicator function. A classical result of Schauder [1] states that the Haar system forms a basis of $L^{p}=L^{p}(0,1), 1 \leq p<\infty$ (with the underlying Lebesgue measure). That is, for every $f \in L^{p}$ there is a unique sequence $a=\left(a_{n}\right)_{n \geq 0}$ of real numbers satisfying $\left\|f-\sum_{k=0}^{n} a_{k} h_{k}\right\|_{L^{p}(0,1)} \rightarrow 0$. For any subset $J$ of nonnegative integers, we will denote by $\mathscr{P}_{J}$ the projection onto the space generated by the subcollection $\left(h_{j}\right)_{j \in J}$. Let $\beta_{p}(h)$ be the unconditional constant of $h$, that is the least $\beta \in[1, \infty]$ such that

$$
\left\|\mathscr{P}_{J} f\right\|_{L^{p}(0,1)} \leq \beta\|f\|_{L^{p}(0,1)},
$$

for any $J \subseteq \mathbb{N}$ and any $f \in L_{p}(0,1)$. Using Paley's inequality [2], Marcinkiewicz [3] proved that $\beta_{p}(h)<\infty$ if and only if $1<p<\infty$. This remarkable and beautiful fact and its various extensions have influenced several areas of mathematics, including the theory singular integrals, stochastic integrals, the structure of Banach spaces, and many others. As an example, let us consider the martingale version of (2), which was obtained by Burkholder in [4]. Assume that $(\Omega, \mathscr{F}, \mathbb{P})$ is a probability space, filtered by $\left(\mathscr{F}_{k}\right)_{k \geq 0}$, a nondecreasing family of sub- $\sigma$-fields of $\mathscr{F}$. Let $f=\left(f_{k}\right)_{k \geq 0}$ be a real-valued martingale with the difference sequence $\left(d f_{k}\right)_{k \geq 0}$ given by $d f_{0}=f_{0}$ and $d f_{k}=f_{k}-f_{k-1}$ for $k \geq 1$. Let $g$ be a transform of $f$ by a predictable sequence $v=\left(v_{k}\right)_{k \geq 0}$ with values in $[0,1]$ : that is, we have $d g_{k}=v_{k} d f_{k}$ for all $k \geq 0$ and by predictability we mean that each term $v_{k}$ is measurable with 
respect to $\mathscr{F}_{(k-1) v 0}$. Then (cf. [4]) for $1<p<\infty$ there is a universal constant $c_{p}^{\prime}$ for which

$$
\|g\|_{p} \leq c_{p}^{\prime}\|f\|_{p}
$$

Here we have used the notation $\|f\|_{p}=\sup _{n}\left\|f_{n}\right\|_{L^{p}(\Omega)}$. Let $c_{p}$ (2) and $c_{p}^{\prime}$ (3) denote the optimal constants in (2) and (3), respectively. The Haar system is a martingale difference sequence with respect to its natural filtration (on the probability space being Lebesgue's unit interval) and hence so is $\left(a_{k} h_{k}\right)_{k \geq 0}$, for given fixed real numbers $a_{0}, a_{1}, a_{2}, \ldots$ (sometimes such special martingales are called Haar martingales, Paley-Walsh martingales, or dyadic martingales). In addition, the deterministic $0-1$ coefficients are allowed in the transforming sequence, so $c_{p}(2) \leq c_{p}^{\prime}$ (3) for all $1<p<\infty$. It follows from the results of Burkholder [5] and Maurey [6] that the constants actually coincide: $c_{p}(2)=c_{p}^{\prime}(3)$ for all $1<p<\infty$. The question about the precise value of $c_{p}(2)$ was answered by Choi in [7]: the description of the constant is quite complicated, so we will not present it here and refer the interested reader to that paper.

Our objective will be to study a certain sharp version of (2), Let us provide some defnitions. Assume that $(M, \mu)$ is a given measure space. A linear (or sublinear) operator $T$ defined on $L^{p}(M)$ and taking values in $L^{p, \infty}(M)$ is said to be of restricted weak type $(p, p)$, if there is a constant $C$ such that, for every measurable set $A \subseteq M$ of finite measure,

$$
\left\|T \chi_{A}\right\|_{L^{p, \infty}(M)} \leq C\left\|\chi_{A}\right\|_{L^{p}(M)} .
$$

Here

$$
\|f\|_{L^{p, \infty}(M)}:=\sup \left\{\lambda^{p} \mu(\{x \in M:|f(x)| \geq \lambda\})\right\}^{1 / p},
$$

is the usual weak quasinorm on the Lorenz space $L^{p, \infty}(M)$. One of the reasons for considering restricted weak-type estimates is that usually these bounds are easier to obtain than other types of inequalities: indeed, the functions involved are bounded and two-valued instead of arbitrary measurable. On the other hand, by means of standard interpolation arguments (see, e.g., Corollary 1.4.21 in Grafakos [8]), a pair of restricted weak-type estimates implies various estimates on intermediate spaces. We will establish a sharp version of restricted weak type bounds for the projections $\mathscr{P}_{J}$. Introduce the constants $C_{p}$ by

$$
C_{p}= \begin{cases}1 & \text { if } 1 \leq p \leq 4 \\ \frac{p}{4} e^{(4-p) / p} & \text { if } p>4\end{cases}
$$

Furthermore, if $g$ is a discrete-time martingale, we define its weak $p$ th quasinorm by $\|g\|_{p, \infty}=\sup _{n}\left\|g_{n}\right\|_{L^{p, \infty}(\Omega)}$. Here is one of our main results.

Theorem 1. Let $f$ be a martingale taking values in $[0,1]$, terminating at $\{0,1\}$ (i.e., satisfying $\lim _{n \rightarrow \infty} f_{n} \in\{0,1\}$ almost surely), and let $g$ be its transform by a predictable sequence with values in $[0,1]$. Then for any $1 \leq p<\infty$ one has

$$
\|g\|_{p, \infty} \leq C_{p}\|f\|_{p} .
$$

The constant $C_{p}$ is the best possible. It is already optimal in the estimate

$$
\left\|\mathscr{P}_{J} \chi_{A}\right\|_{L^{p, \infty}(0,1)} \leq C_{p}\left\|\chi_{A}\right\|_{L^{p}(0,1)},
$$

for the projections associated with the Haar system.

We will also provide a version of this result for the case in which the space $L^{p, \infty}$ is endowed with a different norming. As we will see, this new version of restricted weaktype estimates will be more convenient for applications (cf. Remark 11 below). Namely, for $p>1$ put

$$
\|f\|_{L^{p, \infty}(M)}:=\sup \left\{\frac{1}{\mu(E)^{1-1 / p}} \int_{E}|f| d \mu\right\},
$$

where the supremum is taken over all measurable $E \subseteq M$ with $0<\mu(E)<\infty$. Unfortunately, under these norms, we have managed to prove sharp restricted bounds in the case $p \geq 4$ only (and we do not know the corresponding sharp bounds for $1<p<4$ ). In analogy with the above definitions, if $g$ is a discrete-time martingale, we let \|\|$g \|_{p, \infty}=$ $\left.\sup _{n \geq 0}||\left|g_{n}\right|\right|_{L^{p, \infty}(\Omega)}$.

Theorem 2. Let $f$ be a martingale taking values in $[0,1]$, terminating at $\{0,1\}$, and let $g$ be its transform by a predictable sequence with values in $[0,1]$. Then for any $p \geq 4$ one has

$$
\|g\|_{p, \infty} \leq C_{p}\|f\|_{p} .
$$

The constant $C_{p}$ is the best possible. It is already optimal in the estimate

$$
\left\|\mathscr{P}_{J} \chi_{A}\right\|\left\|_{L^{p, \infty}(0,1)} \leq C_{p}\right\| \chi_{A} \|_{L^{p}(0,1)},
$$

for the projections associated with the Haar system.

So, for $p \geq 4$ the best constant is the same for both norms $\|\cdot\|_{p, \infty}$ and $\||\cdot|\| \|_{p, \infty}$.

All the results discussed above can be formulated in the more general setting of continuous-time martingales. Furthermore, instead of transforms with values in $[0,1]$, one can work under the less restrictive assumption of nonsymmetric differential subordination of martingales (for the necessary definitions and the precise statement of our results, we refer the reader to Section 2). This setting has the advantage of being more convenient for applications, which constitute the second half of the paper. Specifically, we will apply the aforementioned martingale estimates in the study of the corresponding bounds for Fourier multipliers. This will be done in Sections 3 and 4.

\section{A Martingale Inequality}

2.1. Background and Main Results. Assume that $(\Omega, \mathscr{F}, \mathbb{P})$ is a complete probability space, equipped with $\left(\mathscr{F}_{t}\right)_{t \geq 0}$, a nondecreasing family of sub- $\sigma$-fields of $\mathscr{F}$, such that $\mathscr{F}_{0}$ contains all the events of probability 0 . Suppose that $X, Y$ are two adapted real-valued martingales, whose paths are right continuous and have limits from the left. The symbol 
$[X, Y]$ will stand for the quadratic covariance process of $X$ and $Y$ (see, e.g., Dellacherie and Meyer [9] for details). Following Bañuelos and Wang [10] and Wang [11], we say that $Y$ is differentially subordinate to $X$, if the process $\left([X, X]_{t}-[Y, Y]_{t}\right)_{t \geq 0}$ is nonnegative and nondecreasing as a function of $t$. For example, assume that $f$ is a discretetime martingale and let $g$ denote its transform by a certain predictable sequence $v$ with values in $[-1,1]$. Let us treat these two sequences as continuous-time processes, via $X_{t}=f_{|t|}$, $Y_{t}=g_{\lfloor t\rfloor}, t \geq 0$. Then the required condition on $[X, X]-[Y, Y]$ is equivalent to saying that

$$
\left|d g_{k}\right|^{2} \leq\left|d f_{k}\right|^{2}, \quad k=0,1,2, \ldots,
$$

which is the original definition of differential subordination due to Burkholder $[5,12]$. Obviously, this condition is satisfied for the above setting of martingale transforms.

As exhibited in $[13,14]$, martingales $X, Y$ satisfying the differential subordination arise naturally in the martingale study of Fourier multipliers. In this paper, we will work with pairs $X, Y$ satisfying a slightly different condition:

$$
\left([X, Y]_{t}-[Y, Y]_{t}\right)_{t \geq 0} \text { is nondecreasing and nonnegative }
$$

as a function of $t$,

which can be understood as "nonsymmetric differential subordination." For instance, this holds in the above setting of martingale transforms, if we assume that the sequence $v$ takes values in $[0,1]$ (and hence the continuous-time setup does form an extension of the discrete-time case described in the previous section). Inequalities for such martingales were studied by several authors: see, for example, Burkholder [15], Choi [7], and the author [16, 17]. We refer the interested reader to those papers and mention here only result, which will be needed later. It was proven for martingale transforms by Burkholder [15] and in the general continuous-time case by the author in [17]. Throughout, we use the notation $\|X\|_{p}=$ $\sup _{t \geq 0}\left\|X_{t}\right\|_{L^{p}(\Omega)},\|X\|_{p, \infty}=\sup _{t \geq 0}\left\|X_{t}\right\|_{L^{p, \infty}(\Omega)}$, and $\|X X\| \|_{p, \infty}=$ $\left.\sup _{t \geq 0}||\left|X_{t}\right|\right|_{L^{p, \infty}(\Omega)}, 1 \leq p \leq \infty$.

Theorem 3. Let $X, Y$ be two real-valued martingales satisfying (13). Then for any $\lambda>0$ one has

$$
\lambda \sup _{t \geq 0} \mathbb{P}\left(\left|Y_{t}\right| \geq 1\right) \leq\|X\|_{1} .
$$

For each $\lambda$ the inequality is sharp. Therefore, $\|Y\|_{1, \infty} \leq\|X\|_{1}$ and the constant 1 cannot be improved.

We turn our attention to the formulation of the main result of this section. We will use the notation

$$
P(\lambda)= \begin{cases}\lambda^{-1} & \text { if } 0<\lambda \leq 1, \\ e^{4-4 \lambda} & \text { if } \lambda>1\end{cases}
$$

Theorem 4. Suppose that $X$ is a martingale taking values in $[0,1]$ and $Y$ is a real valued martingale such that (13) is satisfied. (i) For any $\lambda>0$ one has

$$
\sup _{t \geq 0} \mathbb{P}\left(\left|Y_{t}\right| \geq \lambda\right) \leq P(\lambda)\|X\|_{1} .
$$

The bound on the right-hand side of (16) is the best possible for each $\lambda$, even in the following version for the Haar system: for any $J \subseteq \mathbb{N}$ and $f:[0,1] \rightarrow[0,1]$,

$$
\left|\left\{x \in[0,1]:\left|\mathscr{P}_{J} f(x)\right| \geq \lambda\right\}\right| \leq P(\lambda)\|f\|_{L^{p}(0,1)} .
$$

(ii) For any $\lambda \geq 1$ one has

$$
4 \sup _{t \geq 0} \mathbb{E}\left(\left|Y_{t}\right|-\lambda+\frac{1}{4}\right)_{+} \leq P(\lambda)\|X\|_{1} .
$$

The bound on the right-hand side is the best possible for each $\lambda$, even in the following version for the Haar system: for any $J \subseteq \mathbb{N}$ and $f:[0,1] \rightarrow[0,1]$,

$$
4\left\|\left(P_{J} f-\lambda+\frac{1}{4}\right)_{+}\right\|_{1} \leq P(\lambda)\|f\|_{L^{p}(0,1)} .
$$

Some comments on the above statement are in order. At the first glance, part (ii) may seem a little artificial, but this is not the case. As we will see (consult Remark 11), the inequality (18) is very convenient for our applications. The second remark concerns the proof of Theorem 4. Namely, the main difficulty lies in showing the assertion for $\lambda>1$. Indeed, when $\lambda \leq 1$, then (16) is an immediate consequence of (14), and its sharpness follows from simple examples. Furthermore, having proved (18) for $\lambda>1$, we deduce the case $\lambda=1$ by a standard limiting argument. Finally, note that $\chi_{\{|y| \geq \lambda\}} \leq 4(|y|-\lambda+1 / 4)_{+}$, which implies that the inequality (18) is stronger than (16). Putting all these facts together, we see that we will be done if we establish the second estimate of Theorem 4 in the case $\lambda>1$ and prove the sharpness of (17) for $\lambda>0$.

2.2. Special Function and Their Properties. The proof of the inequality (18) will be based on Burkholder's method. This technique reduces the problem of proving a given martingale inequality to that of constructing a special function, which possesses certain convexity and majorization properties. For the detailed description of the approach, we refer the interested reader to Burkholder's survey [18] and to the recent monograph [19] by the author.

The purpose of this subsection is to introduce special functions corresponding to (18) and present their basic properties, which will be needed later. We assume that $\lambda>1$ is a fixed parameter. First, consider the following subsets of $[0,1] \times \mathbb{R}:$

$$
\begin{gathered}
D_{1}=\left\{(x, y): \frac{1}{2} x-\frac{1}{4} \leq y \leq x-\frac{1}{4}, y \leq \frac{1}{4}\right\}, \\
D_{2}=\left\{(x, y): x-\frac{1}{4}<y \leq x+\lambda-1, x \leq \frac{1}{2}\right\}, \\
D_{3}=\left\{(x, y): \frac{1}{4} \leq y \leq \lambda-\frac{1}{2}, x>\frac{1}{2}\right\}, \\
D_{4}=\left\{(x, y): y \geq \lambda-\frac{1}{2} \text { or } y \geq x+\lambda-1\right\} .
\end{gathered}
$$


Now we introduce a function $U_{\lambda}$ by

$$
U_{\lambda}(x, y)=\left\{\begin{array}{cc}
e^{3-4 \lambda}\left(4 y^{2}-4 y x\right. & \\
\left.+2 y+\frac{1}{4}\right) & \text { if }(x, y) \in D_{1}, \\
x \exp (-4 x+4 y-4 \lambda+4) & \text { if }(x, y) \in D_{2}, \\
(1-x) \exp (4 y-4 \lambda+2) & \text { if }(x, y) \in D_{3}, \\
(y-x-\lambda)(4 y-4 \lambda+3) & \text { if }(x, y) \in D_{4} \\
+5(y-\lambda)+4 &
\end{array}\right.
$$

and extend it to the whole strip $[0,1] \times \mathbb{R}$ by the condition

$$
\begin{gathered}
U_{\lambda}(x, y)=U_{\lambda}(1-x,-y) \\
\text { for } x \in[0,1], \quad y \in \mathbb{R} .
\end{gathered}
$$

Let us provide some information on this object. In what follows, the symbol $A^{o}$ denotes the interior of a set $A$.

Lemma 5. The function $U_{\lambda}$ enjoys the following properties.

(i) It is of class $C^{1}$ on $(0,1) \times \mathbb{R}$ and of class $C^{\infty}$ in $D_{1}^{o}, D_{2}^{o}$, $D_{3}^{o}$, and $D_{4}^{o}$.

(ii) There is a Borel function c : $D_{1}^{o} \cup D_{2}^{o} \cup D_{3}^{o} \cup D_{4}^{o} \rightarrow$ $[0, \infty)$ with the following property: for any $(x, y) \in$ $D_{1}^{o} \cup D_{2}^{o} \cup D_{3}^{o} \cup D_{4}^{o}$ and any $h, k \in \mathbb{R}$ such that $|x+h| \leq 1$,

$$
\begin{aligned}
& U_{\lambda x x}(x, y) h^{2}+2 U_{\lambda x y}(x, y) h k \\
& \quad+U_{\lambda y y}(x, y) k^{2} \leq c(x, y)\left(k^{2}-h k\right) .
\end{aligned}
$$

(iii) For any $x \in[0,1]$ and $y \in \mathbb{R}$ one has the majorization

$$
U_{\lambda}(x, y) \geq 4\left(|y|-\lambda+\frac{1}{4}\right)_{+} .
$$

(iv) For any $x \in[0,1]$ and $y \in[0, x]$ one has

$$
U_{\lambda}(x, y) \leq x e^{4-4 \lambda}
$$

Proof. (i) This is straightforward. The fact that $U_{\lambda}$ is of class $C^{\infty}$ on each $D_{i}^{o}$ is evident, and to show that $U_{\lambda}$ is of class $C^{1}$ in the strip, one needs to check that the partial derivatives match appropriately at the common boundaries of $D_{1}, D_{2}, D_{3}$, and $D_{4}$. We leave the necessary calculations to the reader.

(ii) If $(x, y) \in D_{1}^{o}$, then the left-hand side of (23) equals $8 e^{3-4 \lambda}\left(k^{2}-h k\right)$, so we may take $c(x, y)=8 e^{3-4 \lambda}$. If $(x, y)$ belongs to the interior of $D_{2}$, the expression on the left of (23) is equal to

$$
\begin{array}{r}
16\left(x-\frac{1}{2}\right)(k-h)^{2} e^{4 y-4 x-4 \lambda+4} \\
+8\left(k^{2}-h k\right) e^{4 y-4 x-4 \lambda+4} .
\end{array}
$$

But $x<1 / 2$, because $(x, y) \in D_{2}^{o}$; therefore $c(x, y)=$ $8 e^{4 y-4 x-4}$ satisfies the desired bound. Next, assume that $(x, y)$ lies in $D_{3}^{o}$. We compute the left-hand side of (23) and obtain

$$
16\left(\frac{1}{2}-x\right) k^{2} e^{4 y-4 \lambda+2}+8\left(h^{2}-h k\right) e^{4 y-4 \lambda+2} .
$$

This time we have $x>1 / 2$ and hence $c(x, y)=8 e^{4 y-4 \lambda+2}$ works fine. Finally, if $(x, y)$ lies in the interior of $D_{4}$, then

$$
\begin{aligned}
& U_{\lambda x x}(x, y) h^{2}+2 U_{\lambda x y}(x, y) h k \\
& \quad+U_{\lambda y y}(x, y) k^{2}=8\left(k^{2}-h k\right),
\end{aligned}
$$

so we may take $c(x, y)=8$.

Before we proceed, let us observe that, by (22), the inequality (23) holds also in the interiors of the "reflected" domains $D_{1}^{\prime}, D_{2}^{\prime}, D_{3}^{\prime}$, and $D_{4}^{\prime}$ given by

$$
D_{i}^{\prime}=\left\{(x, y):(1-x,-y) \in D_{i}\right\},
$$

with $c$ given by $c(1-x,-y)=c(x, y)$.

(iii) Directly from (i) and (ii), the function $U_{\lambda}$ has the following property: for a fixed $y$, the function $x \mapsto U_{\lambda}(x, y)$ is concave on $[0,1]$ (simply plug $k=0$ in (23)). Since the righthand side of (24) does not depend on $x$, it suffices to verify the majorization for $x \in\{0,1\}$ only. Furthermore, because of (22), we may restrict ourselves to two cases $x=0$ and $y \geq-1 / 4 ; x=1$ and $y \geq 1 / 4$. If $x=0$ and $-1 / 4 \leq y \leq \lambda-1 / 4$, then the right-hand side vanishes, while the left-hand side is nonnegative. If $x=0$ and $y>\lambda-1 / 4$, then we must prove that $4(y-\lambda+1)^{2} \geq 4(y-\lambda+1 / 4)$, which is equivalent to the obvious estimate:

$$
(y-\lambda)^{2}+(y-\lambda)+\frac{3}{4} \geq 0
$$

Next, if $x=1$ and $1 / 4 \leq y \leq \lambda-1 / 4$, then both sides of (24) are equal to 0 . Finally, if $x=1$ and $y \geq \lambda-1 / 4$, the majorization reads

$$
4\left(y-\lambda+\frac{1}{2}\right)^{2} \geq 4\left(y-\lambda+\frac{1}{4}\right),
$$

or, equivalently, $(y-\lambda)^{2} \geq 0$.

(iv) Since $U_{\lambda}(0,0)=0$, we can rewrite the bound in the form

$$
U_{\lambda}(x, y)-U_{\lambda}(0,0) \leq x e^{4-4 \lambda} .
$$

It follows from (i) and (ii) that, for any $a \in[0,1]$, the function $\xi_{a}: x \mapsto U_{\lambda}(x, a x)$ is concave (if we put $k=a h$ in (23), the right-hand side of this bound is nonpositive). Consequently, we will be done if we show that $\xi_{a}^{\prime}(0+)$, the onesided derivative of $\xi_{a}$ at 0 , does not exceed $e^{4-4 \lambda}$. But this is simple: we have

$$
\begin{aligned}
\xi_{a}^{\prime}(0+) & =\lim _{d \downarrow 0} \frac{U_{\lambda}(d, a d)}{d} \\
& =\lim _{d \downarrow 0} e^{4 a d-4 d-4 \lambda+4}=e^{4-4 \lambda} .
\end{aligned}
$$

This completes the proof of the lemma. 
2.3. Proof of (18) for $\lambda>1$. It is convenient to split the reasoning into a few separate parts.

Step 1 (a mollification argument). The proof of (18) rests on Itô's formula. Since $U_{\lambda}$ is not of class $C^{2}$, this enforces us to modify $U_{\lambda}$ so that it has the required smoothness. Consider a $C^{\infty}$ function $g: \mathbb{R}^{2} \rightarrow[0, \infty)$, supported on the unit ball of $\mathbb{R}^{2}$ and satisfying $\int_{\mathbb{R}^{2}} g=1$. For a given $\delta \in(0,1 / 4)$, let $U_{\lambda}^{(\delta)}$ be defined on $(\delta, 1-\delta) \times \mathbb{R}$ by the convolution

$$
U_{\lambda}^{(\delta)}(x, y)=\int_{[-1,1]^{2}} U_{\lambda}(x+\delta u, y+\delta v) g(u, v) d u d v .
$$

The function $U_{\lambda}^{(\delta)}$ is of class $C^{\infty}$ in the interior of its domain and inherits the crucial properties from $U_{\lambda}$. Namely, we have the following version of (24):

$$
U_{\lambda}^{(\delta)}(x, y) \geq 4\left(|y|-\lambda+\frac{1}{4}-\delta\right)_{+},
$$

for all $(x, y) \in(\delta, 1-\delta) \times \mathbb{R}$. Next, by Lemma 5 (i) and the integration by parts, we get

$$
U_{\lambda x x}^{(\delta)}(x, y)=\int_{[-1,1]^{2}} U_{\lambda x x}(x+\delta u, y+\delta v) g(u, v) d u d v .
$$

Similar identities hold for $U_{\lambda x y}^{(\delta)}$ and $U_{\lambda y y}^{(\delta)}$, so we see that $U_{\lambda}^{(\delta)}$ satisfies (23) for all $(x, y) \in(\delta, 1-\delta)$, with

$$
c^{(\delta)}(x, y)=\int_{[-1,1]^{2}} c(x+\delta u, y+\delta v) g(u, v) d u d v \geq 0
$$

(the function $c$ constructed above is locally bounded, so there is no problem with the integration).

Step 2 (application of Itô's formula). Take martingales $X, Y$ as in the statement and consider the processes $\bar{X}_{t}=\delta+X_{t} \cdot(1-$ $2 \delta), \bar{Y}_{t}=Y_{t} \cdot(1-2 \delta)$, and $Z_{t}=\left(\bar{X}_{t}, \bar{Y}_{t}\right)$ for $t \geq 0$. Observe that the pair $(\bar{X}, \bar{Y})$ still satisfies (13). Furthermore, $Z$ takes values in the strip $[\delta, 1-\delta] \times \mathbb{R}$, so an application of Itô's formula to the process $\left(U_{\lambda}^{(\delta)}\left(Z_{t}\right)\right)_{t \geq 0}$ yields

$$
U_{\lambda}^{(\delta)}\left(Z_{t}\right)=U_{\lambda}^{(\delta)}\left(Z_{0}\right)+I_{1}+\frac{I_{2}}{2}+I_{3},
$$

where

$$
\begin{aligned}
I_{1}=\int_{0+}^{t} U_{\lambda x}^{(\delta)}\left(Z_{s-}\right) d \bar{X}_{s}+\int_{0+}^{t} U_{\lambda y}^{(\delta)}\left(Z_{s^{-}}\right) d \bar{Y}_{s} \\
I_{2}=\int_{0+}^{t} U_{\lambda x x}^{(\delta)}\left(Z_{s-}\right) d[\bar{X}, \bar{X}]_{s}^{c} \\
+2 \int_{0+}^{t} U_{\lambda x y}^{(\delta)}\left(Z_{s-}\right) d[\bar{X}, \bar{Y}]_{s}^{c} \\
+\int_{0+}^{t} U_{\lambda y y}^{(\delta)}\left(Z_{s^{-}}\right) d[\bar{Y}, \bar{Y}]_{s^{\prime}}^{c}
\end{aligned}
$$

$$
\begin{aligned}
I_{3}=\sum_{0<s \leq t}[ & U_{\lambda}^{(\delta)}\left(Z_{s}\right)-U_{\lambda}^{(\delta)}\left(Z_{s-}\right) \\
& \left.-U_{\lambda x}^{(\delta)}\left(Z_{s-}\right) \Delta \bar{X}_{s}-U_{\lambda y}^{(\delta)}\left(Z_{s-}\right) \Delta \bar{Y}_{s}\right] .
\end{aligned}
$$

Here $\Delta \bar{X}_{s}=\bar{X}_{s}-\bar{X}_{s-}$ denotes the jump of $\bar{X}$ at time $s$, and $[\bar{X}, \bar{X}]^{c}$ is the unique continuous part of the bracket $[\bar{X}, \bar{X}]$ (cf. Dellacherie and Meyer [9]). Let us analyze each of the terms $I_{1}-I_{3}$ separately. We have $\mathbb{E} I_{1}=0$, by the properties of stochastic integrals. By straightforward approximation argument (see, e.g., Wang [11]), the inequality (23) and the domination (13) imply that $I_{2} \leq 0$. Finally, each term in the sum $I_{3}$ is also nonpositive. To see this, observe first that for each $\omega$ we have

$$
\left|\Delta \bar{Y}_{s}(\omega)\right|^{2} \leq \Delta \bar{X}_{s}(\omega) \Delta \bar{Y}_{s}(\omega),
$$

since otherwise the condition (13) would not be satisfied. Now, applying the mean-value property, we get that

$$
\begin{gathered}
U_{\lambda}^{(\delta)}\left(Z_{s}\right)-U_{\lambda}^{(\delta)}\left(Z_{s-}\right) \\
-U_{\lambda x}^{(\delta)}\left(Z_{s^{-}}\right) \Delta \bar{X}_{s}-U_{\lambda y}^{(\delta)}\left(Z_{s-}\right) \Delta \bar{Y}_{s} \\
=\frac{1}{2}\left[U_{\lambda x x}^{(\delta)}(\xi)\left|\Delta \bar{X}_{s}(\omega)\right|^{2}\right. \\
+2 U_{\lambda x y}^{(\delta)}(\xi) \Delta \bar{X}_{s}(\omega) \Delta \bar{Y}_{s}(\omega) \\
\left.+U_{\lambda y y}^{(\delta)}(\xi)\left|\Delta \bar{Y}_{s}(\omega)\right|^{2}\right],
\end{gathered}
$$

where $\xi$ is a certain point in $(\delta, 1-\delta) \times \mathbb{R}$. Using (23), this can be bounded from above by $c^{(\delta)}(\xi)\left[\left|\Delta \bar{Y}_{s}(\omega)\right|^{2}-\right.$ $\Delta \bar{X}_{s}(\omega) \Delta \bar{Y}_{s}(\omega)$ ]. Thus (40) gives $I_{3} \leq 0$.

Step 3 (the final part). If we combine all the above facts and take expectation of both sides of (38), we obtain the estimate $\mathbb{E} U_{\lambda}^{(\delta)}\left(Z_{t}\right) \leq \mathbb{E} U_{\lambda}^{(\delta)}\left(Z_{0}\right)$. By (35), this implies $4 \mathbb{E}\left(\left|\bar{Y}_{t}\right|-\lambda+\right.$ $1 / 4-\delta)_{+} \leq \mathbb{E} U_{\lambda}^{(\delta)}\left(Z_{0}\right)$. If we let $\delta \rightarrow 0$, then $\bar{Y}_{t} \rightarrow Y_{t}$, $Z_{0} \rightarrow\left(X_{0}, Y_{0}\right)$ and $U_{\lambda}^{(\delta)}\left(Z_{0}\right) \rightarrow U_{\lambda}\left(X_{0}, Y_{0}\right)$, so we get

$$
4 \mathbb{E}\left(\left|Y_{t}\right|-\lambda+\frac{1}{4}\right)_{+} \leq \mathbb{E} U_{\lambda}\left(X_{0}, Y_{0}\right),
$$

by Fatou's lemma and Lebesgue's dominated convergence theorem (we have $\left|Z_{0}\right| \leq\left|\bar{X}_{0}\right|+\left|\bar{Y}_{0}\right| \leq 2$ for all $\delta$ ). It remains to use (25): by (13), we have $Y_{0} \in\left[0, X_{0}\right]$ and hence $\mathbb{E} U_{\lambda}\left(X_{0}, Y_{0}\right) \leq P(\lambda) \mathbb{E} X_{0}=P(\lambda)\|X\|_{1}$. Taking the supremum over $t \geq 0$ completes the proof.

2.4. Proofs of Inequalities of Theorems 1 and 2. We start with the following important auxiliary fact.

Corollary 6. Suppose that $X$ is a martingale taking values in $[0,1]$ and let $Y$ be a real-valued martingale such that (13) holds true. Then for any $E \in \mathscr{F}, t \geq 0$, and $\lambda \geq 3 / 4$ one has

$$
\mathbb{E}\left|Y_{t}\right| \chi_{E} \leq \frac{1}{4} e^{3-4 \lambda} \mathbb{E} X_{0}+\lambda \mathbb{P}(E) .
$$


Proof. We have $E=E^{+} \cup E^{-}$, where $E^{+}=E \cap\left\{\left|Y_{t}\right| \geq \lambda\right\}$ and $E^{-}=E \cap\left\{\left|Y_{t}\right|<\lambda\right\}$. By (18),

$$
\begin{aligned}
4 \mathbb{E}\left(\left|Y_{t}\right|-\lambda\right) \chi_{E^{+}} & \leq 4 \mathbb{E}\left(\left|Y_{t}\right|-\left(\lambda+\frac{1}{4}\right)+\frac{1}{4}\right)_{+} \\
& \leq e^{4-4(\lambda+1 / 4)} \mathbb{E} X_{0}=e^{3-4 \lambda} \mathbb{E} X_{0}
\end{aligned}
$$

and, obviously, $4 \mathbb{E}\left(\left|Y_{t}\right|-\lambda\right) \chi_{E^{-}} \leq 0$. Adding the two inequalities above yields the claim.

Equipped with the above statement, we turn to Theorems 1 and 2 .

Proof of (7) and (10). We prove these estimates in the more general continuous-time setting described above. Suppose that $X$ is a martingale taking values in $[0,1]$ and terminating at $\{0,1\}$, and let $Y$ be a real-valued martingale such that (13) is satisfied. Multiplying both sides of (16) by $\lambda^{p}$ gives

$$
\lambda^{p} \sup _{t \geq 0} \mathbb{P}\left(\left|Y_{t}\right| \geq \lambda\right) \leq \begin{cases}\lambda^{p-1}\|X\|_{1} & \text { if } \lambda \leq 1, \\ \lambda^{p} e^{4-4 \lambda}\|X\|_{1} & \text { if } \lambda>1 .\end{cases}
$$

Let us optimize the right-hand side over $\lambda$. If $p<4$, then the maximal value is attained at $\lambda=1$, and we get

$$
\|Y\|_{p, \infty}^{p} \leq\|X\|_{1}=\|X\|_{p}^{p},
$$

where in the last passage we have used the fact that $\lim _{t \rightarrow \infty} X_{t} \in\{0,1\}$. On the other hand, if $p \geq 4$, then the right-hand side of (45) is maximized for the choice $\lambda=$ $p / 4$. Substituting this value of $\lambda$ gives $\|Y\|_{p, \infty}^{p} \leq C_{p}^{p}\|X\|_{1}=$ $C_{p}^{p}\|X\|_{p}^{p}$, and hence (7) follows. The inequality (10) can be proven in a similar manner, with an additional help of (43). Namely, fix appropriate $X, Y$, and $E \in \mathscr{F}$ of positive probability. Assume first that $\mathbb{P}(E) \leq\|X\|_{1}$ and optimize the right-hand side of (43) over $\lambda$. A straightforward analysis of the derivative shows that the maximum is attained for $\lambda=$ $3 / 4+(1 / 4) \log \left(\|X\|_{1} / \mathbb{P}(E)\right) \geq 3 / 4$. Plugging this value of $\lambda$ gives the bound

$$
\begin{aligned}
\mathbb{E}\left|Y_{t}\right| \chi_{E} & \leq \frac{1}{4} \mathbb{P}(E)+\left(\frac{3}{4}+\frac{1}{4} \log \frac{\|X\|_{1}}{\mathbb{P}(E)}\right) \mathbb{P}(E) \\
& =\mathbb{P}(E)^{1 / q}\left[\mathbb{P}(E)^{1 / p}+\frac{1}{4} \mathbb{P}(E)^{1 / p} \log \frac{\|X\|_{1}}{\mathbb{P}(E)}\right]
\end{aligned}
$$

(here $q=p /(p-1)$ is the harmonic conjugate to $p$ ). But the expression in the square brackets, considered as a function of $\mathbb{P}(E)$, does not exceed $C_{p}\left(\|X\|_{1}\right)^{1 / p}$. Thus, it suffices to divide by $\mathbb{P}(E)^{1 / q}$, take the supremum over $E$, and note that $\|X\|_{1}=\|X\|_{p}^{p}$ to get the desired bound. It remains to consider the case when $\mathbb{P}(E)>\|X\|_{1}$. An application of Schwarz inequality, Burkholder's bound $\|Y\|_{2} \leq\|X\|_{2}$ (which follows from the chain $\mathbb{E}\left|Y_{t}\right|^{2}=\mathbb{E}[Y, Y]_{t} \leq \mathbb{E}[X, Y]_{t} \leq$ $\left.\left(\mathbb{E}[Y, Y]_{t}\right)^{1 / 2}\left(\mathbb{E}[X, X]_{t}\right)^{1 / 2} \leq\|Y\|_{2}\|X\|_{2}\right)$, and the fact that $X$ terminates at the set $\{0,1\}$ imply

$$
\begin{aligned}
\mathbb{E}\left|Y_{t}\right| \chi_{E} & \leq\left\|Y_{t}\right\|_{2} \mathbb{P}(E)^{1 / 2} \\
& \leq\|X\|_{2} \mathbb{P}(E)^{1 / 2}=\|X\|_{1}^{1 / 2} \mathbb{P}(E)^{1 / 2} \\
& =\|X\|_{1}^{1 / 2} \mathbb{P}(E)^{1 / 2} .
\end{aligned}
$$

But we have $\|X\|_{1}^{1 / 2} \mathbb{P}(E)^{1 / 2} \leq\|X\|_{1}^{1 / p} \mathbb{P}(E)^{1 / q}$ : indeed, this can be rewritten in the form $\|X\|_{1}^{1 / 2-1 / p} \leq \mathbb{P}(E)^{1 / q-1 / 2}$ and follows from the equality $1 / 2-1 / p=1 / q-1 / 2$ and the bounds $p>4$, $\mathbb{P}(E)>\|X\|_{1}$. Combining this with the above estimate, the inequality $(p / 4) e^{(4-p) / p} \geq 1$, and the equation $\|X\|_{1}=\|X\|_{p}^{p}$, give

$$
\mathbb{E}\left|Y_{t}\right| \chi_{E} \leq C_{p}\|X\|_{p} \mathbb{P}(E)^{1 / q}
$$

Now (10) follows immediately.

2.5. Sharpness of (8), (11), (17), and (19). By an application of the results of Burkholder (see Section 10 in [12]) and Marcinkiewicz [3], the best constants in the inequalities for the Haar system are the same as those in the corresponding estimates for discrete-time martingales (roughly speaking, any martingale pair $(f, g)$, where $g$ is a transform of $f$, can be appropriately embedded into a pair consisting of a dyadic martingale and its transform). This is also closely related to the equality $c_{p}(2)=c_{p}^{\prime}(3)$, which we have discussed at the beginning of the paper. Thus, we will be done if we provide the construction of appropriate martingales.

We start from showing that (17) and (19) are sharp. Assume first that $\lambda \leq 1$, fix $w \in(0, \lambda)$, and define the pair $(f, g)$ by the following conditions:

(i) $f=g$,

(ii) $f_{0} \equiv w \in(0, \lambda)$,

(iii) $f_{1}=f_{2}=\cdots$ is a random variable satisfying

$$
\mathbb{P}\left(f_{1}=0\right)=1-\frac{w}{\lambda}, \quad \mathbb{P}\left(f_{1}=\lambda\right)=\frac{w}{\lambda} .
$$

Then we easily see that $\mathbb{P}\left(\left|g_{1}\right| \geq \lambda\right)=w / \lambda=P(\lambda)\|f\|_{1}$.

We turn to the more difficult case $\lambda>1$. As we have already noted, (19) is stronger than (17), so it suffices to focus on the latter estimate. Let $w \in(0,1 / 2)$ be a fixed number and let $\delta=(\lambda-1) / N$, where $N$ is a large positive integer. Consider a sequence $\left(\xi_{n}\right)_{n=1}^{2 N+1}$ of independent mean-zero random variables with the distribution uniquely determined by the following conditions.

(i) $\xi_{1}$ takes values in $\{-w, \delta-w+1 / 2\}$.

(ii) For $n=1,2, \ldots, N, \xi_{2 n}$ takes values in the set $\{-\delta, 1 / 2-\delta\}$.

(iii) For $n=1,2, \ldots, N-1, \xi_{2 n+1}$ takes values in the set $\{-1 / 2, \delta\}$.

(iv) $\xi_{2 N+1}$ takes values $\pm 1 / 2$.

Next, let $\tau=\inf \left\{n: w+\xi_{1}+\xi_{2}+\cdots+\xi_{n} \in\{0,1\}\right\}$, with the convention $\inf \emptyset=\infty$. It is easy to check that $\tau$ is an almost surely finite stopping time (with respect to the natural filtration of $\xi$ ). Since $\xi_{n}$ are centered, the process

$$
f=\left(f_{n}\right)_{n=0}^{2 N+1}=\left(w+\xi_{1}+\xi_{2}+\cdots+\xi_{\tau \wedge n}\right)_{n=0}^{2 N+1}
$$

is a martingale. Let $g$ denote the transform of $f$ by the deterministic sequence $(1,1,0,1,0,1,0, \ldots)$. To gain some 
intuition about $(f, g)$, let us take a look at its dynamics. The pair starts from the point $(w, w)$ and, at the first move, it goes to $(0,0)$ or to $(1 / 2+\delta, 1 / 2+\delta)$. If it went to $(0,0)$, it stays there forever; if it jumped to $(1 / 2+\delta, 1 / 2+\delta)$, then it moves horizontally either to $(1,1 / 2+\delta)$ (and stops) or to $(1 / 2,1 / 2+$ $\delta)$. If the latter possibility occurs, the movement continues: the pair goes to $(0, \delta)$ (and terminates) or to $(1 / 2+\delta, 1 / 2+2 \delta)$. In the latter case, it moves horizontally to $(1,1 / 2+2 \delta)$ or to $(1 / 2,1 / 2+2 \delta)$ and so on. During the first $2 N$ steps, the pair either hits one of the lines $x=0, x=1$ (and stops) or visits the point $(1 / 2, \lambda-1 / 2)$ on $2 N$ th step. If the latter takes place, then $(f, g)$ jumps to $(0, \lambda-1)$ or to $(1, \lambda)$.

Directly from the above analysis, we see that $f$ takes values in $[0,1]$ and terminates at $\{0,1\}$, and

$$
\begin{aligned}
& \mathbb{P}\left(\left|g_{2 N+1}\right| \geq \lambda\right) \\
&= \mathbb{P}\left(\xi_{1}>0, \xi_{2}<0, \xi_{3}>0,\right. \\
&\left.\xi_{4}<0, \ldots, \xi_{2 N}<0, \xi_{2 N+1}>0\right) \\
&=\frac{w}{1+2 \delta} \cdot\left(\frac{1 / 2-\delta}{1 / 2}\right)^{N}\left(\frac{1 / 2}{1 / 2+\delta}\right)^{N-1} \cdot \frac{1}{2} \\
&=\frac{w}{1+2 \delta}(1-2 \delta)^{N}\left(1-\frac{2 \delta}{1+2 \delta}\right)^{N-1} .
\end{aligned}
$$

However, recall that $\delta=(\lambda-1) / N$. Therefore, if we let $N$ go to infinity, the latter expression converges to $w e^{4-4 \lambda}$. This shows that the constant $P(\lambda)$ cannot be replaced in (17) by a smaller number.

The examples analyzed above can be also used to prove the sharpness of (8) and (11). First, suppose that $p \in[1,4]$ and consider the above example for $\lambda=1$. Then $\|g\|_{p, \infty}^{p} \geq$ $\mathbb{P}\left(\left|g_{1}\right| \geq 1\right)=\|f\|_{1}=\|f\|_{p}^{p}$ and hence $C_{p}=1$ is the best in (8). On the other hand, if $p>4$, we take the above example corresponding to $\lambda=p / 4$ and a large $N$. Then

$$
\begin{aligned}
\|g\|_{p, \infty}^{p} & \geq \lambda^{p} \mathbb{P}\left(\left|g_{2 N+1}\right| \geq \lambda\right) \stackrel{N \rightarrow \infty}{\longrightarrow} \lambda^{p} e^{4-4 \lambda} w \\
& =C_{p}^{p}\|f\|_{1}=C_{p}^{p}\|f\|_{p}^{p}
\end{aligned}
$$

and hence $C_{p}$ cannot be replaced in (8) by a smaller number. This also proves the sharpness of (11), since this bound is stronger than (8) (we easily check that $\|f\|_{p, \infty} \leq\|\| f\|\|_{p, \infty}$ for all $\left.f \in L^{p, \infty}\right)$.

\section{Applications to Fourier Multipliers}

For the sake of convenience, we have split this section into three parts. The first of them contains the necessary definitions, an overview of related facts from the literature and the description of our contribution. The second subsection explains very briefly the martingale representation of a certain class of Fourier multipliers, which will be of importance to us; the material is taken from $[13,14]$, and we have included it here for completeness. The final subsection contains the proof of our main result.
3.1. Background, Notation and Results. It is well known (cf. $[10,13,14,20-23]$ and numerous other papers) that the martingale theory forms an efficient tool to obtain various bounds for many important singular integrals and Fourier multipliers. Recall that, for any bounded function $m: \mathbb{R}^{d} \rightarrow$ $\mathbb{C}$, there is a unique bounded linear operator $T_{m}$ on $L^{2}\left(\mathbb{R}^{d}\right)$, called the Fourier multiplier with the symbol $m$, given by the equality $\widehat{T_{m} f}=m \widehat{f}$. The norm of $T_{m}$ on $L^{2}\left(\mathbb{R}^{d}\right)$ is equal to $\|m\|_{L^{\infty}\left(\mathbb{R}^{d}\right)}$ and a classical problem of harmonic analysis is to study/characterize those $m$, for which the corresponding Fourier multiplier extends to a bounded linear operator on $L^{p}\left(\mathbb{R}^{d}\right), 1<p<\infty$. This question is motivated by the analysis of the classical example, the collection of Riesz transforms $\left\{R_{j}\right\}_{j=1}^{d}$ on $\mathbb{R}^{d}$ (see Stein [24]). Here, for any $j$, the transform $R_{j}$ is a Fourier multiplier corresponding to the $\operatorname{symbol} m(\xi)=$ $-i \xi_{j} /|\xi|, \xi \neq 0$. An alternative definition of $R_{j}$ involves the use of singular integrals:

$$
\begin{aligned}
& R_{j} f(x)=\frac{\Gamma((d+1) / 2)}{\pi^{(d+1) / 2}} \text { p.v. } \int_{\mathbb{R}^{d}} \frac{x_{j}-y_{j}}{|x-y|^{d+1}} f(y) d y, \\
& j=1,2, \ldots, d \text {. }
\end{aligned}
$$

It is well known that singular integral operators play a distinguished role in the theory of partial differential equations and have been used, in particular, in the study of the higher integrability of the gradient of weak solutions. The exact information on the size of such operators (e.g., on the $p$ norms) provides the insight into the degrees of improved regularity and other geometric properties of solutions and their gradients. This gives rise to another classical problem for Fourier multipliers: for a given $m$, provide tight bounds for the size of the multiplier $T_{m}$ in terms of some characteristics of the symbol.

We will extend the aforementioned restricted weak-type estimates to this new setting. We will consider a certain subclass of symbols which are particularly convenient from the probabilistic point of view. Namely, they can be obtained by the modulation of jumps of certain Lévy processes. This class has appeared for the first time in the papers by Bañuelos and Bogdan [13] and Bañuelos et al. [14]. To describe it, let $v$ be a Lévy measure on $\mathbb{R}^{d}$, that is, a nonnegative Borel measure on $\mathbb{R}^{d}$ such that $\nu(\{0\})=0$, and

$$
\int_{\mathbb{R}^{d}} \min \left\{|x|^{2}, 1\right\} \nu(d x)<\infty .
$$

Assume further that $\mu$ is a finite Borel measure on the unit sphere $\mathbb{S}$ of $\mathbb{R}^{d}$ and fix two Borel functions $\phi$ on $\mathbb{R}^{d}$ and $\psi$ on $\mathbb{S}$ which take values in the unit ball of $\mathbb{C}$. We define the associated multiplier $m=m_{\phi, \psi, \mu, \nu}$ on $\mathbb{R}^{d}$ by

$$
\begin{aligned}
m(\xi)=( & \frac{1}{2} \int_{\mathbb{S}}\langle\xi, \theta\rangle^{2} \psi(\theta) \mu(d \theta) \\
& \left.+\int_{\mathbb{R}^{d}}[1-\cos \langle\xi, x\rangle] \phi(x) \nu(d x)\right)
\end{aligned}
$$




$$
\begin{aligned}
& \times\left(\frac{1}{2} \int_{\mathbb{S}}\langle\xi, \theta\rangle^{2} \mu(d \theta)\right. \\
& \left.\quad+\int_{\mathbb{R}^{d}}[1-\cos \langle\xi, x\rangle] \nu(d x)\right)^{-1}
\end{aligned}
$$

if the denominator is not 0 , and $m(\xi)=0$ otherwise. Here $\langle\cdot, \cdot\rangle$ stands for the scalar product in $\mathbb{R}^{d}$. This class includes many important examples, including the real and the imaginary parts of Beurling-Ahlfors operator (cf. $[13,14])$. We will only present here one type of multipliers, which will be of importance later. Pick a proper subset $J$ of $\{1,2, \ldots, d\}$ and take $\mu=\delta_{e_{1}}+\delta_{e_{2}}+\cdots+\delta_{e_{d}}, \nu=0$, and $\psi\left(e_{j}\right)=\chi_{J}(j)$, $j=1,2, \ldots, d$. Here $e_{1}, e_{2}, \ldots, e_{d}$ are the versors in $\mathbb{R}^{d}$. This choice of parameters gives the operator $\sum_{j \in J} R_{j}^{2}$ on $\mathbb{R}^{d}$.

One of the principal results of [14] is the following $L^{p}$ estimate (consult also the earlier paper [25] of Nazarov and Volberg which was devoted to the version for BeurlingAhlfors operator).

Theorem 7. Let $1<p<\infty$ and let $m=m_{\phi, \psi, \mu, \nu}$ be given by (56). Then for any $f \in L^{p}\left(\mathbb{R}^{d}\right)$ one has

$$
\left\|T_{m} f\right\|_{L^{p}\left(\mathbb{R}^{d}\right)} \leq\left(p^{*}-1\right)\|f\|_{L^{p}\left(\mathbb{R}^{d}\right)},
$$

where $p^{*}=\max \{p, p /(p-1)\}$.

It turns out that the constant $p^{*}-1$ is the best possible: see Geiss et al. [22] or Bañuelos and Osȩkowski [26] for details.

Our work will concern a certain subclass of (56), corresponding to those $\phi$ and $\psi$, which take values in $[0,1]$. There are many interesting examples of this type (cf. [26]); for instance the operator $\sum_{j \in J} R_{j}^{2}$ introduced above is of this form. We will prove the following result.

Theorem 8. Suppose that $m$ is a symbol given by (56), where $\phi$ and $\psi$ are assumed to take values in $[0,1]$. Then for any $4 \leq$ $p<\infty$ and any measurable $A \subset \mathbb{R}^{d}$ with $|A|<\infty$,

$$
\left\|\mid T_{m} \chi_{A}\right\|_{L^{p, \infty}\left(\mathbb{R}^{d}\right)} \leq C_{p}\left\|\chi_{A}\right\|_{L^{p}\left(\mathbb{R}^{d}\right)} .
$$

The inequality is sharp. More precisely, for any $4 \leq p<\infty$, any $C<C_{p}$, any $d \geq 2$, and any proper subset $J$ of $\{1,2, \ldots, d\}$ there is $A \subset \mathbb{R}^{d}$ of finite measure such that

$$
\left\|\sum_{j \in J} R_{j}^{2} \chi_{A}\right\|\left\|_{L^{p, \infty}\left(\mathbb{R}^{d}\right)}>C\right\| \chi_{A} \|_{L^{p}\left(\mathbb{R}^{d}\right)} .
$$

Following Stein and Weiss [27], we can give the following application of the above result. Let $T_{m}$ be a Fourier multiplier as in the above statement. Then for any real-valued function $f \in L^{p, 1}\left(\mathbb{R}^{d}\right), p \geq 4$, we have

$$
\left\|\left|T_{m} f\right|\right\|_{L^{p, \infty}\left(\mathbb{R}^{d}\right)} \leq 2^{-1-1 / p} e^{(2-p) / 2}\|f\|_{L^{p, 1}\left(\mathbb{R}^{d}\right)} .
$$

To see this, assume first that $f=\sum_{j=1}^{N} a_{j} \chi_{E_{j}}$, where $a_{1}>a_{2}>$ $\cdots>a_{N}>0$ and $E_{j}$ are pairwise disjoint subsets of $\mathbb{R}^{d}$ of finite measure. Let $F_{0}=\emptyset$ and $F_{j}=E_{1} \cup E_{2} \cup \cdots \cup E_{j}, j=$ $1,2, \ldots, N$. Then $f$ can be rewritten in the form $f=\sum_{j=1}^{N}\left(a_{j}-\right.$ $\left.a_{j+1}\right) \chi_{F_{j}}$, where $a_{N+1}=0$, and

$$
\begin{aligned}
\left\|T_{m} f\right\| \|_{L^{p, \infty}\left(\mathbb{R}^{d}\right)} & \leq\left.\sum_{j=1}^{N}\left(a_{j}-a_{j+1}\right)\left\|T_{m} \chi_{F_{j}}\right\|\right|_{L^{p, \infty}\left(\mathbb{R}^{d}\right)} \\
& \leq \frac{p}{4} e^{(4-p) / p} \sum_{j=1}^{N}\left(a_{j}-a_{j+1}\right)\left\|\chi_{F_{j}}\right\|_{p} \\
& =\frac{p}{4} e^{(4-p) / p} \sum_{j=0}^{N-1} a_{j+1}\left(\left|F_{j+1}\right|^{1 / p}-\left|F_{j}\right|^{1 / p}\right) \\
& =\frac{1}{4} e^{(4-p) / p}\|f\|_{L^{p, 1}\left(\mathbb{R}^{d}\right)} .
\end{aligned}
$$

By standard approximation, the above inequality extends to any nonnegative $f \in L^{p, \infty}\left(\mathbb{R}^{d}\right)$. To pass to general real-valued functions, it suffices to use the decomposition $f=f_{+}-f_{-}$and the inequality $\left\|f_{+}\right\|_{L^{p, 1}\left(\mathbb{R}^{d}\right)}+\left\|f_{-}\right\|_{L^{p, 1}\left(\mathbb{R}^{d}\right)} \leq 2^{1-1 / p}\|f\|_{L^{p, 1}\left(\mathbb{R}^{d}\right)}$.

3.2. The Martingale Representation of the Fourier Multipliers (56). By the reasoning from [14], we are allowed to assume that the Lévy measure $v$ satisfies the symmetry condition $\nu(B)=v(-B)$ for all Borel subsets $B$ of $\mathbb{R}^{d}$. To be more precise, for any $\nu$ there is a symmetric $\bar{\nu}$ which leads to the same multiplier. Furthermore, assume for a while that $|\nu|=$ $\nu\left(\mathbb{R}^{d}\right)$ is finite and nonzero, and introduce the probability measure $\widetilde{v}=\nu /|\nu|$. Consider the independent random variables $T_{-1}, T_{-2}, \ldots, Z_{-1}, Z_{-2}, \ldots$ such that, for each $n=$ $-1,-2, \ldots, T_{n}$ has exponential distribution with parameter $|\nu|$ and $Z_{n}$ takes values in $\mathbb{R}^{d}$ and has $\widetilde{v}$ as the distribution. Next, put $S_{n}=-\left(T_{-1}+T_{-2}+\cdots+T_{n}\right)$ for $n=-1,-2, \ldots$ and let

$$
\begin{gathered}
X_{s, t}=\sum_{s<S_{j} \leq t} Z_{j}, \quad X_{s, t-}=\sum_{s<S_{j}<t} Z_{j}, \\
\Delta X_{s, t}=X_{s, t}-X_{s, t-}
\end{gathered}
$$

for $-\infty<s \leq t \leq 0$. Next, if $f \in L^{\infty}\left(\mathbb{R}^{d}\right)$ is a given function, define its parabolic extension $\mathscr{U}_{f}$ to $(-\infty, 0] \times \mathbb{R}^{d}$ by

$$
\mathcal{U}_{f}(s, x)=\mathbb{E} f\left(x+X_{s, 0}\right) .
$$

Now, fix $x \in \mathbb{R}^{d}, s<0$ and let $f, \phi \in L^{\infty}\left(\mathbb{R}^{d}\right)$. We introduce the processes $F=\left(F_{t}^{x, s, f}\right)_{s \leq t \leq 0}$ and $G=\left(G_{t}^{x, s, f, \phi}\right)_{s \leq t \leq 0}$ by

$$
\begin{gathered}
F_{t}=\mathscr{U}_{f}\left(t, x+X_{s, t}\right), \\
G_{t}=\sum_{s<u \leq t}\left[\Delta F_{u} \cdot \phi\left(\Delta X_{s, u}\right)\right] \\
-\int_{s}^{t} \int_{\mathbb{R}^{d}}\left[\mathcal{U}_{f}\left(v, x+X_{s, v-}+z\right)\right. \\
\left.-\mathscr{U}_{f}\left(v, x+X_{s, v-}\right)\right] \phi(z) v(d z) d v .
\end{gathered}
$$


These processes are martingales adapted to the filtration $\mathscr{F}_{t}=$ $\sigma\left(X_{s, t}: t \in[s, 0]\right)$ (see $\left.[13,14]\right)$. The key fact is the following.

Lemma 9. If $\phi$ takes values in $[0,1]$, then the pair $\left(F^{x, s, f}\right.$, $G^{x, s, f, \phi}$ ) satisfies (13).

Proof. The assertion follows immediately from the identities

$$
\begin{gathered}
{[F, G]_{t}=\sum_{s<u \leq t}\left|\Delta F_{u}\right|^{2} \phi\left(\Delta X_{s, u}\right),} \\
{[G, G]_{t}=\sum_{s<u \leq t}\left|\Delta F_{u}\right|^{2}\left(\phi\left(\Delta X_{s, u}\right)\right)^{2},}
\end{gathered}
$$

which can be established by repeating the reasoning from [13].

Now we introduce a family of multipliers. Fix $s<0$, a function $\phi$ on $\mathbb{R}^{d}$ taking values in the unit ball of $\mathbb{C}$, and define the operator $\mathscr{T}=\mathscr{T}^{s}$ by the bilinear form:

$$
\int_{\mathbb{R}^{d}} \mathscr{T} f(x) g(x) d x=\int_{\mathbb{R}^{d}} \mathbb{E}\left[G_{0}^{x, s, f, \phi} g\left(x+X_{s, 0}\right)\right] d x,
$$

where $f, g \in C_{0}^{\infty}\left(\mathbb{R}^{d}\right)$. We have the following fact, proven in [13].

Lemma 10. Let $1<p<\infty$ and $d \geq 2$. The operator $\mathscr{T}^{s}$ is well defined and extends to a bounded operator on $L^{p}\left(\mathbb{R}^{d}\right)$, which can be expressed as a Fourier multiplier with the symbol

$$
\begin{aligned}
M(\xi)= & M_{s}(\xi) \\
= & {\left[1-\exp \left(2 s \int_{\mathbb{R}^{d}}(1-\cos \langle\xi, z\rangle) \nu(d z)\right)\right] } \\
& \times \frac{\int_{\mathbb{R}^{d}}(1-\cos \langle\xi, z\rangle) \phi(z) \nu(d z)}{\int_{\mathbb{R}^{d}}(1-\cos \langle\xi, z\rangle) \nu(d z)}
\end{aligned}
$$

if $\int_{\mathbb{R}^{d}}(1-\cos \langle\xi, z\rangle) \nu(d z) \neq 0$, and $M(\xi)=0$ otherwise. Furthermore, (66) holds true for all $f \in C_{0}^{\infty}\left(\mathbb{R}^{d}\right)$ and all $g$ belonging to $L^{q}\left(\mathbb{R}^{d}\right)$ for some $1<q<\infty$.

3.3. Proof of (58). We may and do assume that at least one of the measures $\mu, v$ is nonzero. It is convenient to split the reasoning into two parts.

Step 1. First we show the estimate for the multipliers of the form

$$
M_{\phi, v}(\xi)=\frac{\int_{\mathbb{R}^{d}}(1-\cos \langle\xi, z\rangle) \phi(z) \nu(d z)}{\int_{\mathbb{R}^{d}}(1-\cos \langle\xi, z\rangle) \nu(d z)} .
$$

Assume that $0<\nu\left(\mathbb{R}^{d}\right)<\infty$, so that the above machinery using Lévy processes is applicable. Fix $s<0$ and functions $f, g \in C_{0}^{\infty}\left(\mathbb{R}^{d}\right)$ such that $f$ takes values in $[0,1]$, while $g$ takes values in $[-1,1]$ and is supported on a certain set $E$ of finite Lebesgue measure. Of course, then the martingale $F^{x, s, f}$ takes values in $[0,1]$. By Fubini's theorem and (43), for any $\lambda \geq 3 / 4$ we have

$$
\begin{aligned}
& \left|\int_{\mathbb{R}^{d}} \mathbb{E}\left[G_{0}^{x, s, f, \phi} g\left(x+X_{s, 0}\right)\right] d x\right| \\
& \leq \int_{\mathbb{R}^{d}} \mathbb{E}\left|G_{0}^{x, s, f, \phi}\right| \chi_{\left\{x+X_{s, 0} \in E\right\}} d x \\
& \leq \frac{e^{3-4 \lambda}}{4} \int_{\mathbb{R}^{d}} \mathbb{E}\left|F_{0}^{x, s, f}\right| d x \\
& \quad+\lambda \int_{\mathbb{R}^{d}} \mathbb{P}\left(x+X_{s, 0} \in E\right) d x \\
& =\frac{e^{3-4 \lambda}}{2}\|f\|_{L^{1}\left(\mathbb{R}^{d}\right)}+\lambda|E| .
\end{aligned}
$$

Plugging this into the definition of $\mathcal{S}$ and taking the supremum over all $g$ as above, we obtain

$$
\int_{E}\left|\mathcal{S}^{s, \phi, v} f(x)\right| d x \leq \frac{e^{3-4 \lambda}}{2}\|f\|_{L^{1}\left(\mathbb{R}^{d}\right)}+\lambda|E| .
$$

Now if we let $s \rightarrow-\infty$, then $M_{s, \phi, \nu}$ converges pointwise to the multiplier $M_{\phi, \nu}$ given by (68). By Plancherel's theorem, $\delta^{s, \phi, v} f \rightarrow T_{M_{\phi, v}} f$ in $L^{2}\left(\mathbb{R}^{d}\right)$ and hence there is a sequence $\left(s_{n}\right)_{n=1}^{\infty}$ converging to $-\infty$ such that $\lim _{n \rightarrow \infty} \mathcal{S}^{s_{n}, \phi, v} f \rightarrow$ $T_{M_{\phi, \nu}} f$ almost everywhere. Thus Fatou's lemma combined with (70) yields the bound

$$
\int_{E}\left|T_{M_{\phi, \nu}} f(x)\right| d x \leq \frac{e^{3-4 \lambda}}{4}\|f\|_{L^{1}\left(\mathbb{R}^{d}\right)}+\lambda|E| .
$$

Now we repeat word by word the optimization arguments used in Section 2 in the proof of (10) (we need to consider the cases $\|f\|_{L^{1}\left(\mathbb{R}^{d}\right)} \leq|E|$ and $\|f\|_{L^{1}\left(\mathbb{R}^{d}\right)}>|E|$ separately). As the result, we obtain the bound

$$
\int_{E}\left|T_{M_{\phi, v}} f(x)\right| d x \leq C_{p}\|f\|_{L^{1}\left(\mathbb{R}^{d}\right)}^{1 / p}|E|^{1 / q} .
$$

Finally, using some standard approximation arguments, we see that (72) can be applied to $f=\chi_{A}$ (where $A$ is a measurable subset of $\mathbb{R}^{d}$, satisfying $|A|<\infty$ ), and we get the estimate:

$$
\left\|T_{M_{\phi, v}} \chi_{A}\right\|_{L^{p, \infty}\left(\mathbb{R}^{d}\right)} \leq C_{p}\left\|\chi_{A}\right\|_{L^{p}\left(\mathbb{R}^{d}\right)} .
$$

This is precisely the desired claim (but for the above special multipliers).

Step 2. Now we deduce the result for the general multipliers as in (56) and drop the assumption $0<v\left(\mathbb{R}^{d}\right)<\infty$. For a given $\varepsilon>0$, define a Lévy measure $v_{\varepsilon}$ in polar coordinates $(r, \theta) \in(0, \infty) \times \mathbb{S}$ by

$$
\nu_{\varepsilon}(d r d \theta)=\varepsilon^{-2} \delta_{\varepsilon}(d r) \mu(d \theta) .
$$

Here $\delta_{\varepsilon}$ denotes Dirac measure on $\{\varepsilon\}$. Next, consider a multiplier $M_{\varepsilon, \phi, \psi, \mu, \nu}$ as in (68), in which the Lévy measure is 
$\chi_{\{|x|>\varepsilon\}} \nu+\nu_{\varepsilon}$ and the jump modulator is given by $\chi_{\{|x|>\varepsilon\}} \phi(x)+$ $\chi_{\{|x|=\varepsilon\}} \psi(x /|x|)$. Note that this Lévy measure is finite and nonzero, at least for sufficiently small $\varepsilon$. If we let $\varepsilon \rightarrow 0$, we see that

$$
\begin{aligned}
\int_{\mathbb{R}^{d}}[ & 1-\cos \langle\xi, x\rangle] \psi\left(\frac{x}{|x|}\right) \nu_{\varepsilon}(d x) \\
& =\int_{\mathbb{S}}\langle\xi, \theta\rangle^{2} \phi(\theta) \frac{1-\cos \langle\xi, \varepsilon \theta\rangle}{\langle\xi, \varepsilon \theta\rangle^{2}} \mu(d \theta) \\
& \longrightarrow \frac{1}{2} \int_{\mathbb{S}}\langle\xi, \theta\rangle^{2} \phi(\theta) \mu(d \theta)
\end{aligned}
$$

and, consequently, $M_{\varepsilon, \phi, \psi, \mu, \nu} \rightarrow m_{\phi, \psi, \mu, \nu}$ pointwise. Thus (73) yields (7). Indeed, using Plancherel's theorem as above, we see that there is a sequence $\left(\varepsilon_{n}\right)_{n \geq 1}$ converging to 0 such that $T_{M_{\varepsilon_{n}, \phi, \psi}, \nu} \chi_{A} \rightarrow T_{m_{\phi, \psi, \mu, \nu}} \chi_{A}$ almost everywhere. It suffices to apply Fatou's lemma, and the proof is complete.

Remark 11. An important comment is in order. The above proof rests on the estimate (43), which we have managed to prove in the case $\lambda \geq 3 / 4$ only; this is the reason why the restricted bound (58) holds only for $p \geq 4$. To get a sharp bound for $p<4$, we would require a version of (43) for small $\lambda$; unfortunately, the bound (16) does not seem to be powerful enough to yield any result of this type.

\section{On the Lower Bound for the Constant in (58)}

We turn to the final section of the paper in which we will show that the constant $C_{p}$ in (58) is the best possible. The proof will be a combination of various analytic and probabilistic facts, and it is convenient to split the reasoning into a several separate parts. Throughout this section, $\mathscr{B} \subset \mathbb{C}$ denotes the ball of center 0 and radius 1 .

4.1. Laminates: Necessary Definitions. Assume that $\mathbb{R}^{m \times n}$ denotes the space of all real matrices of dimension $m \times n$ and let $\mathbb{R}_{\text {sym }}^{n \times n}$ be the subclass of $\mathbb{R}^{n \times n}$ which consists of all real symmetric $n \times n$ matrices.

Definition 12. A function $f: \mathbb{R}^{m \times n} \rightarrow \mathbb{R}$ is said to be rankone convex, if $t \mapsto f(A+t B)$ is convex for all $A, B \in \mathbb{R}^{m \times n}$ with $\operatorname{rank} B=1$.

Let $\mathscr{P}=\mathscr{P}\left(\mathbb{R}^{m \times n}\right)$ stand for the class of all compactly supported probability measures on $\mathbb{R}^{m \times n}$. For $v \in \mathscr{P}$, we denote by $\bar{\nu}=\int_{\mathbb{R}^{m \times n}} X d \nu(X)$ the center of mass or barycenter of $\nu$.

Definition 13. We say that a measure $\nu \in \mathscr{P}$ is a laminate (and write $v \in \mathscr{L}$ ), if

$$
f(\bar{\nu}) \leq \int_{\mathbb{R}^{m \times n}} f d \nu
$$

for all rank-one convex functions $f$. The set of laminates with barycenter 0 is denoted by $\mathscr{L}_{0}\left(\mathbb{R}^{m \times n}\right)$.
Laminates can be used to obtain lower bounds for solutions of certain PDEs, as was first noticed by Faraco in [28]. Furthermore, laminates arise naturally in several applications of convex integration, where they can be used to produce interesting counterexamples; see, for example, [29-33]. We will be particularly interested in the case of $2 \times 2$ symmetric matrices. The important fact is that laminates can be regarded as probability measures that record the distribution of the gradients of smooth maps; see Corollary 17. Let us briefly explain this; detailed proofs of the statements below can be found, for example, in [32-34].

Definition 14. Let $U \subset \mathbb{R}^{2 \times 2}$ be a given set. Then $\mathscr{P} \mathscr{L}(U)$ denotes the class of prelaminates in $U$, that is, the smallest class of probability measures on $U$ which

(i) contains all measures of the form $\lambda \delta_{A}+(1-\lambda) \delta_{B}$ with $\lambda \in[0,1]$ and satisfying $\operatorname{rank}(A-B)=1$;

(ii) is closed under splitting in the following sense: if $\lambda \delta_{A}+(1-\lambda) v$ belongs to $\mathscr{P} \mathscr{L}(U)$ for some $v \in$ $\mathscr{P}\left(\mathbb{R}^{2 \times 2}\right)$ and $\mu$ also belongs to $\mathscr{P} \mathscr{L}(U)$ with $\bar{\mu}=A$, then also $\lambda \mu+(1-\lambda) \nu$ belongs to $\mathscr{P} \mathscr{L}(U)$.

By the successive application of Jensen's inequality, we have the inclusion $\mathscr{P} \mathscr{L} \subset \mathscr{L}$. Let us state two well-known facts (see $[29,32-34])$.

Lemma 15. Let $v=\sum_{i=1}^{N} \lambda_{i} \delta_{A_{i}} \in \mathscr{P} \mathscr{L}\left(\mathbb{R}_{\text {sym }}^{2 \times 2}\right)$ with $\bar{v}=0$. Moreover, let $0<r<(1 / 2) \min \left|A_{i}-A_{j}\right|$ and $\delta>0$. For any bounded domain $\Omega \subset \mathbb{R}^{2}$ there exists $u \in W_{0}^{2, \infty}(\Omega)$ such that $\|u\|_{C^{1}}<\delta$ and for all $i=1 \cdots N$

$$
\left|\left\{x \in \Omega:\left|D^{2} u(x)-A_{i}\right|<r\right\}\right|=\lambda_{i}|\Omega| .
$$

Lemma 16. Let $K \subset \mathbb{R}_{\text {sym }}^{2 \times 2}$ be a compact convex set and $\nu \in$ $\mathscr{L}\left(\mathbb{R}_{\text {sym }}^{2 \times 2}\right)$ with supp $v \subset K$. For any relatively open set $U \subset$ $\mathbb{R}_{\text {sym }}^{2 \times 2}$ with $K \subset \subset U$ there exists a sequence $v_{j} \in \mathscr{P} \mathscr{L}(U)$ of prelaminates with $\bar{\nu}_{j}=\bar{v}$ and $\nu_{j} \stackrel{*}{\rightarrow} \nu$.

These two lemmas, combined with a simple mollification, yield the following statement proven originally by Boros et al. [35]. It exhibits the connection between laminates supported on symmetric matrices and second derivatives of functions and will play a crucial role below.

Corollary 17. Let $v \in \mathscr{L}_{0}\left(\mathbb{R}_{\text {sym }}^{2 \times 2}\right)$. Then there exists a sequence $u_{j} \in C_{0}^{\infty}(\mathscr{B})$ with uniformly bounded second derivatives, such that

$$
\frac{1}{|\mathscr{B}|} \int_{\mathscr{B}} \phi\left(D^{2} u_{j}(x)\right) d x \longrightarrow \int_{\mathbb{R}_{s y m}^{2 \times 2}} \phi d \nu
$$

for all continuous $\phi: \mathbb{R}_{\text {sym }}^{2 \times 2} \rightarrow \mathbb{R}$.

Let us stress here that the corollary works for laminates of barycenter 0 . This will give rise to some small technical difficulties, as "natural" laminates do not have this property; see below. 
4.2. Biconvex Functions and a Special Laminate. In the next step in our analysis, we introduce a certain special laminate. To do this, we need some additional notation. A function $\zeta: \mathbb{R} \times \mathbb{R} \rightarrow \mathbb{R}$ is said to be biconvex if, for any fixed $z \in \mathbb{R}$, the functions $x \mapsto \zeta(x, z)$ and $y \mapsto \zeta(z, y)$ are convex. Now, for a given $p \geq 4$, pick $\lambda=p / 4$ and let $f, g$ be martingales of Section 2, which exhibit the sharpness of (10) and (11) (actually, there is a whole family of examples, corresponding to different choices of $w$ and $N$-these two parameters will be specified later). Consider the $\mathbb{R}^{2}$-valued martingale:

$$
(F, G):=(g-w, f-g) .
$$

We subtract $w$ on the first coordinate to ensure that the pair $(F, G)$ has mean $(0,0)$. This sequence has the following zigzag property: for any $0 \leq n \leq 2 N+1$ we have $F_{n}=F_{n+1}$ with probability 1 or $G_{n}=G_{n+1}$ almost surely; that is, in each step $(F, G)$ moves either horizontally or vertically. Indeed, this follows directly from the construction that for each $n$ we have $\mathbb{P}\left(d f_{n}=d g_{n}\right)=1$ or $\mathbb{P}\left(d g_{n}=0\right)=1$. This property combines nicely with biconvex functions: if $\zeta$ is such a function, then a successive application of Jensen's inequality gives

$$
\begin{aligned}
& \mathbb{E} \zeta\left(F_{2 N+2}, G_{2 N+2}\right) \\
& \quad \geq \mathbb{E} \zeta\left(F_{2 N+1}, G_{2 N+1}\right) \geq \cdots \geq \mathbb{E} \zeta\left(F_{0}, G_{0}\right)=\zeta(0,0)
\end{aligned}
$$

Now, the martingale $(F, G)$, or rather the distribution of its terminal variable $\left(F_{2 N+2}, G_{2 N+2}\right)$, gives rise to a probability measure $v$ on $\mathbb{R}_{\text {sym }}^{2 \times 2}$ : put

$$
\begin{array}{r}
\nu(\operatorname{diag}(x, y))=\mathbb{P}\left(\left(F_{2 N+2}, G_{2 N+2}\right)=(x, y)\right), \\
(x, y) \in \mathbb{R}^{2} .
\end{array}
$$

Here and below, $\operatorname{diag}(x, y)$ denotes the diagonal matrix $\left(\begin{array}{ll}x & 0 \\ 0 & y\end{array}\right)$. The key observation is that $\nu$ is a laminate of barycenter 0 . To prove this, note that if $\psi: \mathbb{R}^{2 \times 2}$ is a rank-one convex, then $(x, y) \mapsto \psi(\operatorname{diag}(x, y))$ is biconvex and thus, by $(80)$,

$$
\begin{aligned}
\int_{\mathbb{R}^{2 \times 2}} \psi d \nu & =E \psi\left(\operatorname{diag}\left(F_{2 N+2}, G_{2 N+2}\right)\right) \\
& \geq \psi(\operatorname{diag}(0,0))=\psi(\bar{\nu}) .
\end{aligned}
$$

Finally, note that $\mathbb{P}\left(F_{2 N+2}+G_{2 N+2} \in\{-w, 1-w\}\right)=$ $\mathbb{P}\left(f_{2 N+2} \in\{0,1\}\right)=1$, and hence the support of $\nu$ is contained in

$$
K=\{\operatorname{diag}(x, y): x+y \in\{-w, 1-w\}\}
$$

4.3. Sharpness of (58): The Case $d=2$. We will prove that $C_{p}$ is the best in

$$
\left\|R_{1}^{2} \chi_{A}\right\|_{L^{p, \infty}\left(\mathbb{R}^{d}\right)} \leq C_{p}\left\|\chi_{A}\right\|_{L^{p}\left(\mathbb{R}^{d}\right)} .
$$

For the convenience of the reader, let us first sketch the idea. We start with the application Corollary 17 to the laminate $v$ : let $\left(u_{j}\right)_{j \geq 1}$ be the corresponding sequence of smooth functions. As we have just observed above, the support of $v$ is contained in $K$ given by (83). Since the distribution of $u_{j}$ is close to $v$ (in the sense of Corollary 17), we expect that $\Delta u_{j}$, essentially, takes only values close to $-w$ or close to $1-w$. Thus, if we define $v_{j}=\Delta u_{j}+w \chi_{\mathscr{B}}$ for $j=1,2, \ldots$, then $v_{j}$ is close to an indicator function of a certain set $A$. Thus, to prove the sharpness of (84), one can try to study this estimate with $\chi_{A}$ replaced by $v_{j}$. We will look separately at the action on $R_{1}^{2}$ on $\Delta u_{j}$ and $w \chi_{\mathscr{B}}$; to handle the Laplacian, we will use the arguments from the previous two subsections, and $w \chi_{\mathscr{B}}$ will be dealt with the aid of (57).

Step 1. We start from the specification of the parameters $N$ and $w$. For a given $p \geq 4$, pick an arbitrary number $M$ smaller than $e^{4-4 \lambda} / 4$ (recall that $\lambda=p / 4$ ): thus, $M=(1 / 4) e^{4-4 \lambda} \cdot \eta$ for some $\eta<1$. Let $w \in(0,1 / 2)$ be arbitrary and choose $N$ so that $\mathbb{E}\left(\left|g_{2 N+2}\right|-\lambda\right)_{+}>M \mathbb{E} f_{0}=M w$. This is possible, in view of the results of Section 2. Furthermore, let $\varepsilon$ be an arbitrary positive number (which will eventually be sent to 0 ). In what follows, we will use the following convention: $C_{1}, C_{2}, C_{3}, \ldots$ will denote constants which depend only on $w$ and $N$.

Step 2. Consider a continuous function $\phi: \mathbb{R}_{\text {sym }}^{2 \times 2} \rightarrow \mathbb{R}$ given by $\phi(\operatorname{diag}(x, y))=|x+y+w|$. By Corollary 17, we have

$$
\begin{aligned}
\frac{1}{|\mathscr{B}|} \int_{\mathscr{B}}\left|v_{j}\right| & =\frac{1}{|\mathscr{B}|} \int_{\mathscr{B}} \phi\left(D^{2} u_{j}\right) \stackrel{j \rightarrow \infty}{\longrightarrow} \int_{\mathbb{R}_{\mathrm{sym}}^{2 \times 2}} \phi d \nu \\
& =\mathbb{E}\left|F_{2 N+2}+G_{2 N+2}+w\right|=w,
\end{aligned}
$$

so for sufficiently large $j$ we have

$$
\frac{1}{|\mathscr{B}|} \int_{\mathscr{B}}\left|v_{j}\right| \leq w(1+\varepsilon)
$$

Step 3. Consider a continuous function $\phi: \mathbb{R}_{\text {sym }}^{2 \times 2} \rightarrow[0,1]$, which satisfies $\phi(\operatorname{diag}(x, y))=0$ if $x+y+w \in\{0,1\}$ and which is 1 if the distance between $x+y+w$ and the set $\{0,1\}$ is larger than $\varepsilon$. By Corollary 17 ,

$$
\frac{1}{|\mathscr{B}|} \int_{\mathscr{B}} \phi\left(D^{2} u_{j}\right) \longrightarrow \int_{\mathbb{R}_{\text {sym }}^{2 \times 2}} \phi d v=0,
$$

since $\mathbb{P}\left(F_{2 N+2}+G_{2 N+2}+w \in\{0,1\}\right)=1$. Consider the sets

$$
\begin{gathered}
A=\left\{x \in \mathscr{B}:\left|\Delta u_{j}(x)-1+w\right| \leq \varepsilon\right\}, \\
\widetilde{A}=\left\{x \in \mathscr{B}:\left|\Delta u_{j}(x)+w\right| \leq \varepsilon\right\} .
\end{gathered}
$$

Then (87) implies that

$$
\frac{|\mathscr{B} \backslash(A \cup \widetilde{A})|}{|\mathscr{B}|}<\varepsilon \quad \text { for sufficiently large } j .
$$

Step 4. Next, consider a continuous function $\phi: \mathbb{R}_{\text {sym }}^{2 \times 2} \rightarrow$ $[0,1]$, satisfying $\phi(\operatorname{diag}(x, y))=1$ if $x+y+w=1$ and $\phi(\operatorname{diag}(x, y))=0$ if $|x+y+w-1|>\varepsilon$. Then

$$
\begin{aligned}
|A| & \geq \int_{\mathscr{B}} \phi\left(D^{2} u_{j}\right) \stackrel{j \rightarrow \infty}{\longrightarrow}|\mathscr{B}| \int_{\mathbb{R}_{\text {sym }}^{2 \times 2}} \phi d v \\
& =|\mathscr{B}| \mathbb{P}\left(F_{2 N+2}+G_{2 N+2}=1-w\right)=|\mathscr{B}| w .
\end{aligned}
$$


Thus, for large $j$,

$|A|$ is bounded from below by $C_{1}$.

Consequently, for any $1 \leq q<\infty$ and large $j$,

$$
\begin{aligned}
\| v_{j}- & \chi_{A} \|_{L^{q}\left(\mathbb{R}^{2}\right)}^{q} \\
= & \left\|\Delta u_{j}+w-\chi_{A}\right\|_{L^{q}(\mathscr{B})}^{q} \\
= & \int_{A}\left|\Delta u_{j}+w-\chi_{A}\right|^{q}+\int_{\widetilde{A}}\left|\Delta u_{j}+w-\chi_{A}\right|^{q} \\
& +\int_{\mathscr{B} \backslash(A \cup \widetilde{A})}\left|\Delta u_{j}+w-\chi_{A}\right|^{q} \\
\leq & \varepsilon^{q}|A|+\varepsilon^{q}|\widetilde{A}|+\varepsilon|\mathscr{B}|\left(\sup _{\mathscr{B}}\left|\Delta u_{j}\right|+w\right) .
\end{aligned}
$$

Here in the last passage we have used the definition of $A$, $\widetilde{A}$, and (89). Combining this with (91) (and the fact that the second-order partial derivatives of $u_{j}$ are uniformly bounded by $C_{2}$; see Corollary 17), we get that, for sufficiently large $j$,

$$
\left\|v_{j}-\chi_{A}\right\|_{L^{q}\left(\mathbb{R}^{2}\right)}^{q} \leq C_{3} \varepsilon|A| \text {. }
$$

In other words, the function $v_{j}$ is close to the indicator function of $A$.

Step 5. Next, consider the function $\phi: \mathbb{R}_{\mathrm{sym}}^{2 \times 2} \rightarrow \mathbb{R}$ given by $\phi(\operatorname{diag}(x, y))=(|x+w|-\lambda)_{+}$. By the choice of $w, N$, and (86),

$$
\begin{aligned}
\frac{1}{|\mathscr{B}|} \int_{\mathscr{B}} \phi\left(D^{2} u_{j}\right) & \stackrel{j \rightarrow \infty}{\longrightarrow} \int_{\mathbb{R}_{\text {sym }}^{2 \times 2}} \phi d v=\mathbb{E}\left(\left|g_{2 N+2}\right|-\lambda\right)_{+} \\
& >M w \geq \frac{M}{1+\varepsilon} \cdot \frac{1}{|\mathscr{B}|} \int_{\mathscr{B}}\left|v_{j}\right| \\
& \geq \frac{M}{1+\varepsilon} \cdot \frac{1}{|\mathscr{B}|}\left(|A|-\int_{\mathscr{B}}\left|v_{j}-\chi_{A}\right|\right) .
\end{aligned}
$$

Now multiply throughout by $|\mathscr{B}|$ and apply (93) with $q=1$; we get that, for sufficiently large $j$,

$$
\int_{\mathscr{B}} \phi\left(D^{2} u_{j}\right) \geq \frac{M}{1+\varepsilon}\left(1-C_{3} \varepsilon\right)|A| .
$$

However, observe that

$$
\begin{aligned}
\phi\left(D^{2} u_{j}\right) & =\left(\left|\partial_{11} u_{j}+w\right|-\lambda\right)_{+} \\
& =\left(\left|R_{1}^{2} \Delta u_{j}+w\right|-\lambda\right)_{+} \\
& =\left(\left|R_{1}^{2} v_{j}-w R_{1}^{2} \chi_{\mathscr{B}}+w\right|-\lambda\right)_{+}
\end{aligned}
$$

on $\mathscr{B}$. Therefore, the preceding considerations yield that, for large $j$,

$$
\begin{aligned}
\frac{M}{1+\varepsilon}\left(1-C_{3} \varepsilon\right)|A| \leq & \int_{\mathscr{B}}\left(\left|R_{1}^{2} v_{j}-w R_{1}^{2} \chi_{\mathscr{B}}+w\right|-\lambda\right)_{+} \\
\leq & \int_{\mathscr{B}}\left(\left|R_{1}^{2} \chi_{A}-w R_{1}^{2} \chi_{\mathscr{B}}+w\right|-\lambda\right)_{+} \\
& +\int_{\mathscr{B}}\left|R_{1}^{2}\left(v_{j}-\chi_{A}\right)\right| .
\end{aligned}
$$

However, the norm of $R_{1}^{2}$ as an operator on $L^{2}\left(\mathbb{R}^{2}\right)$ is bounded by 1: see (57). Consequently, by Schwarz inequality, (93), and then (91),

$$
\begin{aligned}
\int_{\mathscr{B}}\left|R_{1}^{2}\left(v_{j}-\chi_{A}\right)\right| & \leq\left\|v_{j}-\chi_{A}\right\|_{2}|\mathscr{B}|^{1 / 2} \\
& \leq C_{4} \varepsilon^{1 / 2}|A|^{1 / 2} \leq C_{5} \varepsilon^{1 / 2}|A| .
\end{aligned}
$$

Plugging this into the above inequality, we get that if $j$ is sufficiently large, then

$$
\int_{\mathscr{B}}\left(\left|R_{1}^{2} \chi_{A}-w R_{1}^{2} \chi_{\mathscr{B}}+w\right|-\lambda\right)_{+} \geq \frac{M}{1+\varepsilon}\left(1-C_{6} \varepsilon^{1 / 2}\right)|A| .
$$

Therefore, if we let $E=\left\{x \in \mathscr{B}:\left|R_{1}^{2} \chi_{A}-w R_{1}^{2} \chi_{\mathscr{B}}+w\right|>\lambda\right\}$ and recall that $M=(1 / 4) e^{4-4 \lambda} \cdot \eta$, then

$$
\begin{aligned}
\int_{E}\left|R_{1}^{2} \chi_{A}-w R_{1}^{2} \chi_{\mathscr{B}}+w\right| \\
\geq \frac{M}{1+\varepsilon}\left(1-C_{6} \varepsilon^{1 / 2}\right)|A|+\lambda|E| \\
\geq \frac{\left(1-C_{6} \varepsilon^{1 / 2}\right) \eta}{1+\varepsilon}\left[\frac{e^{4-4 \lambda}}{4}|A|+\lambda|E|\right] .
\end{aligned}
$$

However, we have $\lambda=p / 4$. Plugging this above and applying the Young inequality, we see that the right-hand side is not smaller than

$$
\frac{\left(1-C_{6} \varepsilon^{1 / 2}\right) \eta}{1+\varepsilon} \cdot \frac{p}{4} e^{(4-p) / p}\left\|\chi_{A}\right\|_{L^{p}\left(\mathbb{R}^{2}\right)}|E|^{1-1 / p} .
$$

On the other hand, $\left\|R_{1}^{2}\right\|_{L^{p}\left(\mathbb{R}^{2}\right) \rightarrow L^{p}\left(\mathbb{R}^{2}\right)} \leq p-1$ (see (57)), so by the Schwarz inequality and the bound $|A| \geq w$ (see the estimate above (91),

$$
\begin{aligned}
& \int_{E}\left|w R_{1}^{2} \chi_{\mathscr{B}}-w\right| \\
& \leq w\left(|E|+(p-1)|\mathscr{B}|^{1 / p}|E|^{1-1 / p}\right) \\
& \leq w^{1-1 / p}|A|^{1 / p} \\
& \times\left(|E|+(p-1)|\mathscr{B}|^{1 / p}|E|^{1-1 / p}\right) \\
& \leq p \pi^{1 / p} w^{1-1 / p}\left\|\chi_{A}\right\|_{L^{p}\left(\mathbb{R}^{2}\right)}|E|^{1-1 / p} .
\end{aligned}
$$

Combining this with the previous estimate, we get

$$
\begin{aligned}
\int_{E}\left|R_{1}^{2} \chi_{A}\right| & \\
\geq & \left(\frac{\left(1-C_{6} \varepsilon^{1 / 2}\right) \eta}{1+\varepsilon} \cdot \frac{p}{4} e^{(4-p) / p}-p \pi^{1 / p} w^{1-1 / p}\right) \\
& \times\left\|\chi_{A}\right\|_{L^{p}\left(\mathbb{R}^{2}\right)}|E|^{1-1 / p} .
\end{aligned}
$$

Using the fact that $\eta\langle 1, \varepsilon>0$ and $w>0$ were arbitrary, we obtain that the constant $(p / 4) e^{(4-p) / p}$ is indeed the best possible in (58). 
4.4. Sharpness of (58): The Case $d \geq 3$. Let $J$ be a proper subset of $\{1,2, \ldots, d\}$ and write $T=\sum_{j \in J} R_{j}^{2}$. It suffices to consider only those $J$, which satisfy $1 \in J$ and $2 \notin J$ : for any $J^{\prime} \in\{1,2, \ldots, d\}$ of the same cardinality as $J$, the restricted weak constants of $T$ and $\sum_{j \in J^{\prime}} R_{j}^{2}$ are the same. So, suppose that $T$ is of that special form and assume that for some positive constant $C$ we have

$$
\int_{E}\left|R_{2}^{2} \chi_{A}(x)\right| d x \leq C\left\|\chi_{A}\right\|_{L^{p}\left(\mathbb{R}^{d}\right)}|E|^{1-1 / p}
$$

for all measurable subsets $A, E$ of $\mathbb{R}^{d}$ of positive and finite Lebesgue measure. For $t>0$, define the dilation operator $\delta_{t}$ as follows: for any function $g: \mathbb{R}^{2} \times \mathbb{R}^{d-2} \rightarrow \mathbb{R}$, we let $\delta_{t} g(\xi, \zeta)=g(\xi, t \zeta)$; for any $A \subset \mathbb{R}^{2} \times \mathbb{R}^{d-2}$, let $\delta_{t} A=\{(\xi, t \zeta)$ : $(\xi, \zeta) \in A\}$. Note that the function $\delta_{t} \chi_{A}$ is supported on $\delta_{t}^{-1} A$ and hence, by (104), the operator $T_{t}:=\delta_{t}^{-1} \circ R_{1}^{2} \circ \delta_{t}$ satisfies

$$
\begin{aligned}
\int_{E}\left|T_{t} \chi_{A}(x)\right| d x= & t^{d-2} \int_{\delta_{t}^{-1} E}\left|R_{1}^{2} \circ \delta_{t} \chi_{A}(x)\right| d x \\
\leq & C\left(t^{d-2} \int_{\delta_{t}^{-1} A}\left|\delta_{t} \chi_{A}(x)\right|^{p} d x\right)^{1 / p} \\
& \times\left(t^{d-2}\left|\delta_{t}^{-1} E\right|\right)^{1-1 / p} \\
= & C\left\|\chi_{A}\right\|_{L^{p}\left(\mathbb{R}^{d}\right)}|E|^{1-1 / p} .
\end{aligned}
$$

It is straightforward to check that the Fourier transform $\mathscr{F}$ satisfies the identity $\mathscr{F}=t^{d-2} \delta_{t} \circ \mathscr{F} \circ \delta_{t}$; since $1 \in J$ and $2 \notin J$, the operator $T_{t}$ has the property that

$$
\begin{array}{r}
\widehat{T_{t} f}(\xi, \zeta)=-\frac{\xi_{1}^{2}+t^{2} \sum_{j \in K} \zeta_{j}^{2}}{|\xi|^{2}+t^{2}|\zeta|^{2}} \widehat{f}(\xi, \zeta), \\
(\xi, \zeta) \in \mathbb{R}^{2} \times \mathbb{R}^{d-2},
\end{array}
$$

where the set $K$ is defined by the requirement that $k \in K$ if and only if $k+2 \in J$. By Lebesgue's dominated convergence theorem, we have

$$
\lim _{t \rightarrow 0} \widehat{T_{t} f}(\xi, \zeta)=\widehat{T_{0} f}(\xi, \zeta)
$$

in $L^{2}\left(\mathbb{R}^{d}\right)$, where $\widehat{T_{0} f}(\xi, \zeta)=-\xi_{1}^{2} \widehat{f}(\xi, \zeta) /|\xi|^{2}$. By Plancherel's theorem, the passage to a subsequence which converges almost everywhere, and Fatou's lemma, we see that (105) implies

$$
\int_{E}\left|T_{0} \chi_{A}(x)\right| d x \leq C\left\|\chi_{A}\right\|_{L^{p}\left(\mathbb{R}^{d}\right)}|E|^{1-1 / p} .
$$

Now pick an arbitrary set $\widetilde{A} \subset \mathbb{R}^{2}$ of nonzero and finite Lebesgue measure and put $A=\widetilde{A} \times[0,1]^{d-2}$. Denoting by $\mathscr{R}_{1}$ the first planar Riesz transform, we see that $T_{0} \chi_{A}(\xi, \zeta)=$ $\mathscr{R}_{1}^{2} \chi_{\widetilde{A}}(\xi) \chi_{[0,1]^{d-2}}(\zeta)$, because of the identity

$$
\widehat{T_{0} \chi_{A}}(\xi, \zeta)=-\frac{\xi_{1}^{2}}{|\xi|^{2}} \widehat{\chi}_{\widetilde{A}}(\xi) \widehat{\chi_{[0,1]^{d-2}}}(\zeta)
$$

Plug this into (108) with the choice $E=\widetilde{E} \times[0,1]^{d-2}$, where $\widetilde{E}$ is an arbitrary subset of $\mathbb{R}^{2}$ with $0<|\widetilde{E}|<\infty$. As the result, we obtain

$$
\int_{\widetilde{E}}\left|\mathscr{R}_{1}^{2} \chi_{\widetilde{A}}(\xi)\right| d \xi \leq C\left\|\chi_{\widetilde{A}}\right\|_{L^{p}\left(\mathbb{R}^{2}\right)}|\widetilde{E}|^{1-1 / p} .
$$

But we have shown in the previous subsection that this implies $C \geq p e^{(4-p) / p} / 4$. The proof is complete.

\section{Acknowledgments}

The author would like to thank the anonymous referee for the careful reading of the paper. The research was partially supported by Polish Ministry of Science and Higher Education (MNiSW) Grant IP2011 039571 “Iuventus Plus.”

\section{References}

[1] J. Schauder, "Eine Eigenschaft des Haarschen Orthogonalsystems," Mathematische Zeitschrift, vol. 28, no. 1, pp. 317-320, 1928.

[2] R. E. A. C. Paley, "A remarkable series of orthogonal functions (I)," Proceedings of the London Mathematical Society, vol. 34, no. 241, p. 264, 1932.

[3] J. Marcinkiewicz, "Quelques théorèmes sur les séries orthogonales," Annales de la Société Polonaise de Mathématique, vol. 16, pp. 84-96, 1937.

[4] D. L. Burkholder, "Martingale transforms," Annals of Mathematical Statistics, vol. 37, pp. 1494-1504, 1966.

[5] D. L. Burkholder, "A geometrical characterization of Banach spaces in which martingale difference sequences are unconditional," The Annals of Probability, vol. 9, no. 6, pp. 997-1011, 1981.

[6] B. Maurey, "Système de Haar," in Séminaire Maurey-Schwartz 1974-1975, École Polytechnique, Paris, France, 1975.

[7] K. P. Choi, "A sharp inequality for martingale transforms and the unconditional basis constant of a monotone basis in $L^{p}(0,1)$," Transactions of the American Mathematical Society, vol. 330, no. 2, pp. 509-529, 1992.

[8] L. Grafakos, Classical Fourier Analysis, vol. 249 of Graduate Texts in Mathematics, Springer, New York, NY, USA, 2nd edition, 2008.

[9] C. Dellacherie and P.-A. Meyer, Probabilities and Potential B: Theory of Martingales, vol. 72 of North-Holland Mathematics Studies, North-Holland, Amsterdam, The Netherlands, 1982.

[10] R. Bañuelos and G. Wang, "Sharp inequalities for martingales with applications to the Beurling-Ahlfors and Riesz transforms," Duke Mathematical Journal, vol. 80, no. 3, pp. 575-600, 1995.

[11] G. Wang, "Differential subordination and strong differential subordination for continuous-time martingales and related sharp inequalities," The Annals of Probability, vol. 23, no. 2, pp. 522-551, 1995.

[12] D. L. Burkholder, "Boundary value problems and sharp inequalities for martingale transforms," The Annals of Probability, vol. 12, no. 3, pp. 647-702, 1984.

[13] R. Bañuelos and K. Bogdan, "Lévy processes and Fourier multipliers," Journal of Functional Analysis, vol. 250, no. 1, pp. 197-213, 2007.

[14] R. Bañuelos, A. Bielaszewski, and K. Bogdan, "Fourier multipliers for non-symmetric Lévy processes," in Marcinkiewicz Centenary Volume, vol. 95 of Banach Center Publications, pp. 925, Polish Academy of Sciences, Warsaw, Poland, 2011. 
[15] D. L. Burkholder, "An extension of a classical martingale inequality," in Probability Theory and Harmonic Analysis (Cleveland, Ohio, 1983), vol. 98 of Monographs and Textbooks in Pure and Applied Mathematics, pp. 21-30, Dekker, New York, NY, USA, 1986.

[16] A. Osękowski, "Logarithmic estimates for nonsymmetric martingale transforms," Statistics and Probability Letters, vol. 80, no. 7-8, pp. 678-682, 2010.

[17] A. Osękowski, "Sharp weak type inequalities for the Haar system and related estimates for nonsymmetric martingale transforms," Proceedings of the American Mathematical Society, vol. 140, no. 7, pp. 2513-2526, 2012.

[18] D. L. Burkholder, "Explorations in martingale theory and its applications," in École d'Été de Probabilités de Saint-Flour XIX1989, vol. 1464 of Lecture Notes in Mathematics, pp. 1-66, Springer, Berlin, Germany, 1991.

[19] A. Osękowski, Sharp Martingale and Semimartingale Inequalities, vol. 72 of Monografie Matematyczne, Birkhäuser, Basel, Switzerland, 2012.

[20] R. Bañuelos and P. Janakiraman, " $L^{p}$-bounds for the BeurlingAhlfors transform," Transactions of the American Mathematical Society, vol. 360, no. 7, pp. 3603-3612, 2008.

[21] R. Bañuelos and P. J. Méndez-Hernández, "Space-time Brownian motion and the Beurling-Ahlfors transform," Indiana University Mathematics Journal, vol. 52, no. 4, pp. 981-990, 2003.

[22] S. Geiss, S. Montgomery-Smith, and E. Saksman, "On singular integral and martingale transforms," Transactions of the American Mathematical Society, vol. 362, no. 2, pp. 553-575, 2010.

[23] A. Osękowski, "Logarithmic inequalities for Fourier multipliers," Mathematische Zeitschrift, vol. 274, no. 1-2, pp. 515-530, 2013.

[24] E. M. Stein, Singular Integrals and Differentiability Properties of Functions, Princeton Mathematical Series, Princeton University Press, Princeton, NJ, USA, 1970.

[25] F. Nazarov and A. Volberg, "Heat extension of the Beurling operator and estimates for its norm," St. Petersburg Mathematical Journal, vol. 15, pp. 563-573, 2004.

[26] R. Bañuelos and A. Osȩkowski, "Martingales and sharp bounds for Fourier multipliers," Annales Academice Scientiarum Fennica, Mathematica, vol. 37, no. 1, pp. 251-263, 2012.

[27] E. M. Stein and G. Weiss, "An extension of a theorem of Marcinkiewicz and some of its applications," Journal of Mathematics and Mechanics, vol. 8, pp. 263-284, 1959.

[28] D. Faraco, "Milton's conjecture on the regularity of solutions to isotropic equations," Annales de l'Institut Henri Poincaré, Analyse Non Linéaire, vol. 20, no. 5, pp. 889-909, 2003.

[29] K. Astala, D. Faraco, and L. Székelyhidi, Jr., "Convex integration and the $L^{p}$ theory of elliptic equations," Annali della Scuola Normale Superiore di Pisa, Classe di Scienze 5, vol. 7, no. 1, pp. $1-50,2008$.

[30] S. Conti, D. Faraco, and F. Maggi, "A new approach to counterexamples to $L^{1}$ estimates: Korn's inequality, geometric rigidity, and regularity for gradients of separately convex functions," Archive for Rational Mechanics and Analysis, vol. 175, no. 2, pp. 287-300, 2005.

[31] B. Kirchheim, S. Müller, and V. Šverák, "Studying nonlinear pde by geometry in matrix space," in Geometric Analysis and Nonlinear Partial Differential Equations, pp. 347-395, Springer, Berlin, Germany, 2003.

[32] S. Müller and V. Šverák, "Convex integration for Lipschitz mappings and counterexamples to regularity," Annals of Mathematics 2, vol. 157, no. 3, pp. 715-742, 2003.
[33] L. Székelyhidi Jr., "Counterexamples to elliptic regularity and convex integration," Contemporary Mathematics, vol. 424, pp. 227-245, 2007.

[34] B. Kirchheim, Rigidity and geometry of microstructures [Habilitation Thesis], University of Leipzig, 2003, http://www.mis.mpg .de/publications/other-series/ln/lecturenote-1603.html.

[35] N. Boros, L. Székelyhidi Jr., and A. Volberg, "Laminates meet Burkholder functions," Journal de Mathématiques Pures et Appliquées, vol. 100, no. 5, pp. 687-700, 2013. 


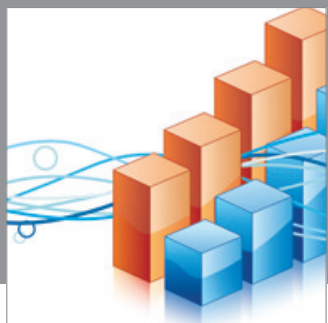

Advances in

Operations Research

mansans

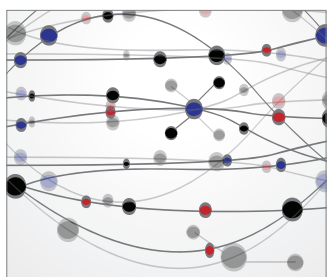

The Scientific World Journal
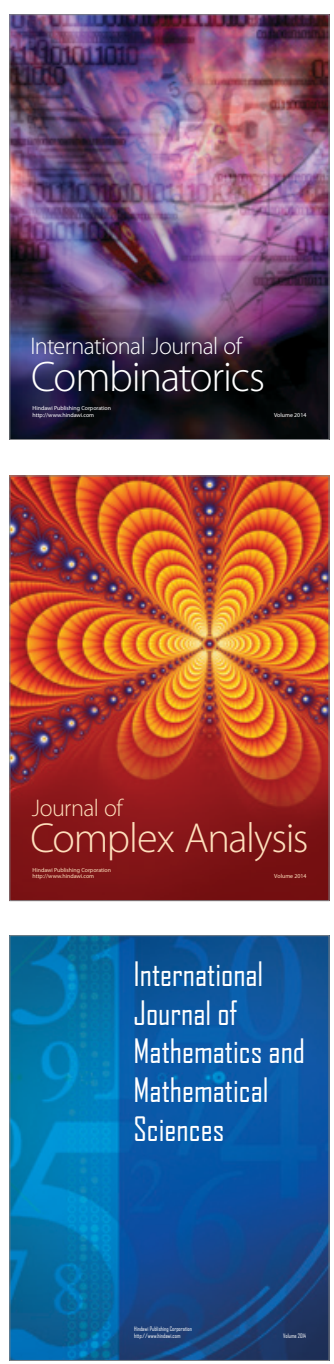
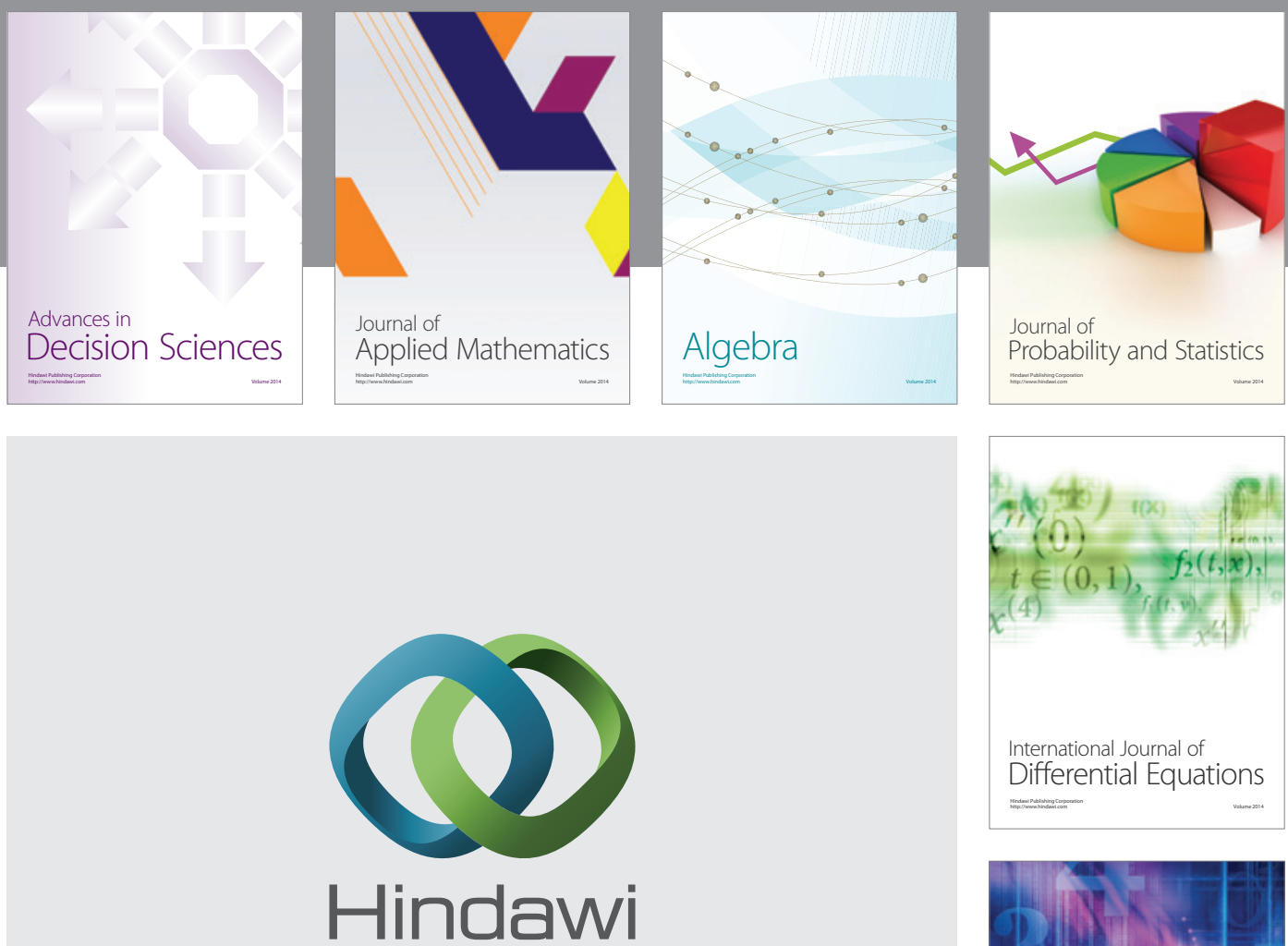

Submit your manuscripts at http://www.hindawi.com
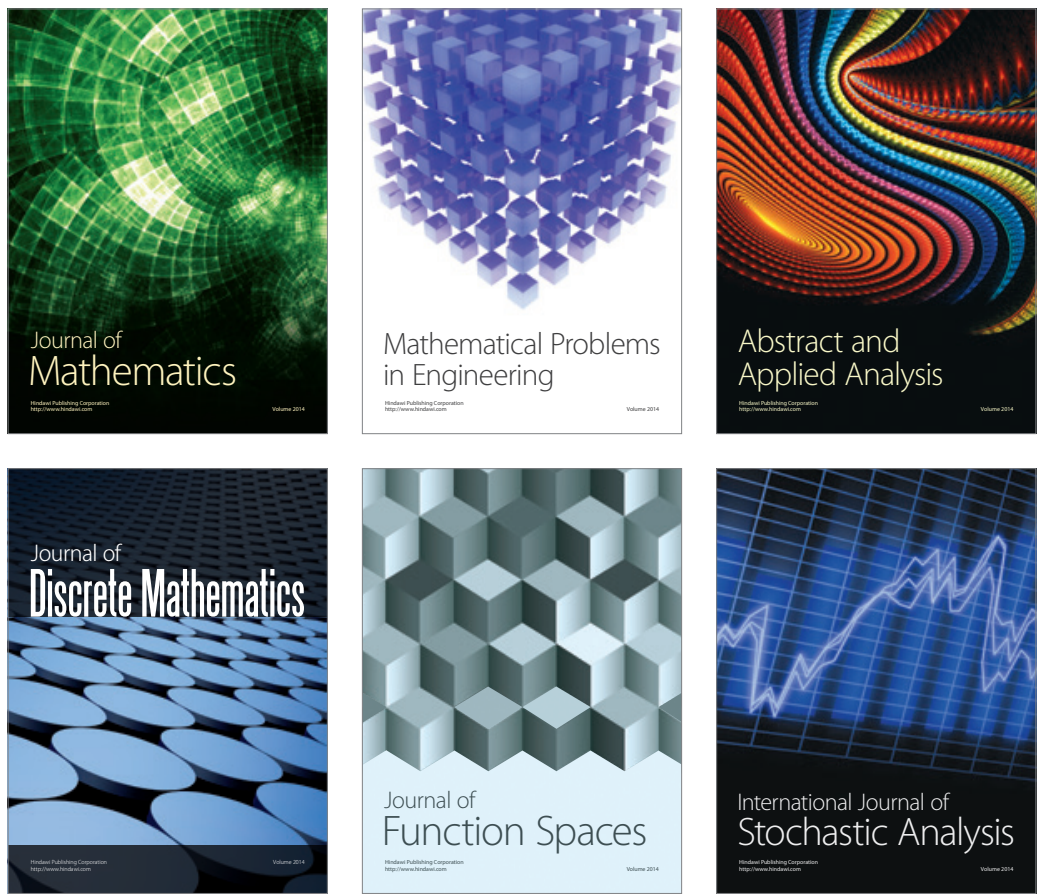

Journal of

Function Spaces

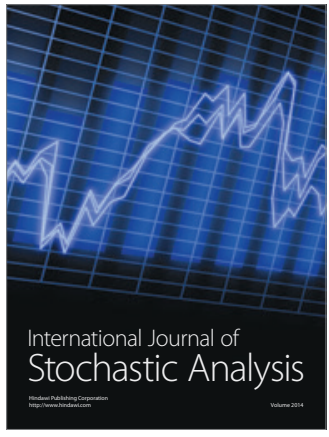

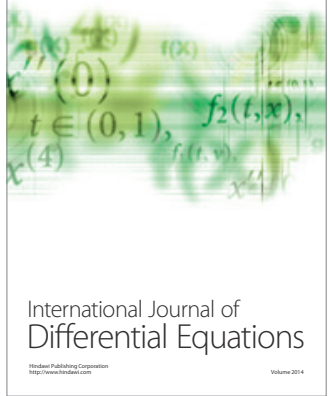
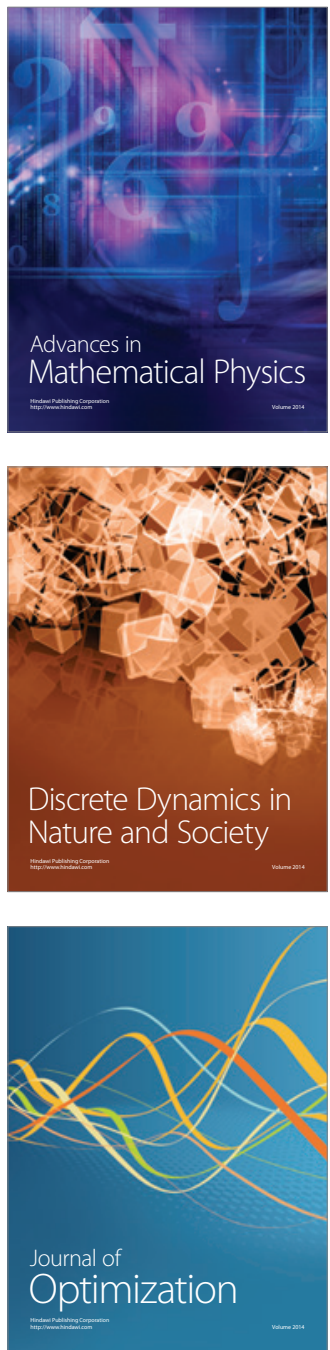